\title{
HIGH-RESOLUTION PHOTOELECTRON SPECTROSCOPY OF MOLECULES
}

\author{
Kwanghsi Wang and Vincent McKoy \\ Arthur Amos Noyes Laboratory of Chemical Physics, ${ }^{1}$ California Institute \\ of Technology, Pasadena, California 91125
}

KEY WORDS: REMPI, ZEKE, Cooper minima, parity selection rules, photoelectron matrix elements

\begin{abstract}
Rotationally resolved photoelectron spectra can provide significant insight into the underlying dynamics of molecular photoionization. Here, we discuss and compare results of recent theoretical studies of rotationally resolved photoelectron spectra with measurements for molecules such as $\mathrm{HBr}, \mathrm{OH}, \mathrm{NO}, \mathrm{N}_{2}, \mathrm{CO}, \mathrm{H}_{2} \mathrm{O}, \mathrm{H}_{2} \mathrm{CO}$, and $\mathrm{CH}_{3}$. These studies reveal the rich dynamics of quantum-state-specific studies of molecular photoionization and provide a robust description of key spectral features resulting from Cooper minima, autoionization, alignment, partial-wave mixing, and interference in related experimental studies.
\end{abstract}

\section{INTRODUCTION}

Rotationally resolved photoelectron spectra can provide significant insight into the underlying dynamics of molecular photoionization, one of the simplest of molecular fragmentation processes. As in all fragmentation processes, determination of the final state distributions of the fragments provides a window on the electronic and nuclear motion of the collision complex responsible for the asymptotic fragment distributions. In photoionization, the collision complex consists of the photoelectron and ion

${ }^{1}$ Contribution No. 9036. 
core at short range, and rotationally resolved photoelectron spectra hence serve as a probe of electron-core interactions at short range. In addition to providing such fundamental insights, these quantum-state-specific photoelectron spectra have clear implications for studies of intramolecular relaxation processes, of elemental clusters, of weakly bound molecular complexes, and of state-selected ion-molecule reactions (1).

Conventional photoelectron analyzers have been used to obtain rotationally resolved photoelectron spectra for light systems such as $\mathrm{H}_{2}$ $(2-4), \mathrm{D}_{2}(5,6)$ and for high- $J$ levels of excited electronic states of NO (79), $\mathrm{NH}(10,11), \mathrm{OH}(12)$, and SH (13), where the large rotational spacings make such measurements possible. These resonance-enhanced multiphoton ionization (REMPI) studies of $\mathrm{NO}, \mathrm{NH}, \mathrm{OH}$, and $\mathrm{SH}$ (7-13) have, in fact, provided rotationally resolved photoelectron spectra for single rotational levels of excited electronic states. With the recent development of zero-kinetic-energy (ZEKE) photoelectron spectroscopy (1, 14-18), based on the detection of photoelectrons produced by delayed pulsed-field ionization (PFI) of high Rydberg states lying below an ion threshold, however, it is now possible to exploit the narrow bandwidth of laser radiation to routinely achieve subwavenumber resolution in cation distributions. With its unprecedented resolution, this technique has already found many new and exciting applications in physical chemistry (14). Laser-induced-fluorescence (LIF) detection of photoions can provide even higher resolution than energy-resolved photoelectron studies and has been used to determine the populations of individual $\Lambda$ components of rotational levels of the ion (19).

To date, studies of rotationally resolved photoelectron spectra have mainly focused on the threshold or near-threshold regions. Extension of such studies to higher photoelectron energies can provide valuable insight into the dynamics of molecular photoionization. Constraints on the energy range accessible with lasers and resolution limits for high-energy electron detection restrict photoelectron studies to the near-threshold region. However, detection of dispersed fluorescence from excited photoions can provide ion rotational distributions deep into the ionization continuum (20). Because fluorescence is observed, the detection bandwidth is uncoupled from, and therefore not limited by, the excitation bandwidth, thus making it possible to exploit the broad tunability of synchrotron radiation. This strategy significantly expands the range of possible studies into photoionization dynamics. For example, ion rotational distributions for photoionization of $\mathrm{N}_{2}$ and $\mathrm{CO}$ have been measured for photoelectron energies up to $200 \mathrm{eV}$ above threshold (20). These studies demonstrated that the energy dependence of the ion rotational distributions can indeed extend to high photoelectron energies and that the photoions retained significant 
alignment, implying that the molecular character of the ionization dynamics persists at such high energies.

REMPI, coupled with high-resolution photoelectron spectroscopy, and ZEKE photoelectron spectroscopy have been used to obtain rotationally resolved photoelectron spectra for a wide range of molecules (1-18). These rotationally resolved studies have highlighted significant spectral features, such as the dependence of photoelectron angular distributions on rotational levels $(4,9)$, rotational propensity rules $(8,9,18,19)$, parity selectivity in transitions involving electronically degenerate states $(3,8$, 18 ), autoionization $(6,21,22)$, effects of alignment $(23)$, and the influence of Cooper minima $(10-12,20)$ and shape resonances $(20,24)$ on ion distributions. On the other hand, progress in related theoretical studies of such spectra has been more limited (25-30). A quantitative description of the underlying dynamics of rotationally resolved photoelectron spectra can clearly be expected to present new theoretical and computational challenges.

In this article we review the progress we have made in our theoretical studies of rotationally resolved photoelectron spectra of several diatomic and small polyatomic molecules. We choose not to give an exhaustive review of all the systems and features we have studied. Instead, after a brief review of the theoretical framework and computational approach employed in these studies, we discuss a few selected examples that illustrate central underlying dynamical issues in rotationally resolved photoelectron spectra, such as strong $\ell$ mixing in molecular photoelectron continua, parity selection rules, and Cooper minima. These examples include spectra at low photoelectron energies resulting from $(2+1)$ REMPI of single rotational levels of $\mathrm{HBr}$ and $\mathrm{OH}$; ZEKE photoelectron spectra for singlephoton ionization of jet-cooled ground state $\mathrm{NO}, \mathrm{CO}, \mathrm{H}_{2} \mathrm{O}, \mathrm{H}_{2} \mathrm{CO}$, and $\mathrm{CH}_{3}$ by coherent VUV radiation; and $\mathrm{N}_{2}$ and $\mathrm{CO}$ ion distributions at very high photoelectron energies that have been studied by dispersed fluorescence from excited photoions (20). These studies highlight the rich underlying dynamics of quantum-state-specific molecular photoionization and provide a robust description of key spectral features in the measured spectra.

\section{THEORY AND FORMULATION}

\section{$\left(n+1^{\prime}\right)$ REMPI for Linear Molecules}

ROTATIONALLY RESOLVED PHOTOELECTRON SPECTRA The general theory of molecular photoionization processes used in these studies has been discussed previously (31). Here we present just a brief outline of some essential features of our approach, as used to obtain rotationally resolved photo- 


\section{8} WANG \& McKOY

electron spectra. Where appropriate, we note conditions that apply to single-photon ionization. In an $\left(n+1^{\prime}\right)$ REMPI process, the resonant intermediate state is created by absorption of $n$ photons from an initially unaligned (all $M_{J_{0}}$ levels equally populated) state. This aligned state (levels with different $\left|M_{J_{i}}\right|$ values have different populations) is subsequently ionized by absorption of another photon. The photon energies of the excitation and ionization steps of REMPI may differ from each other in a two-color experiment. Under collision-free conditions, ionization out of each $M_{J_{0}}$ sublevel of the initial state forms an independent channel for linearly polarized light. The differential cross section for photoionization of the resonant state by a single photon can then be written as

$$
\frac{d \sigma}{d \Omega}=\frac{\sigma}{4 \pi}\left[1+\sum_{L=1}^{n+1} \beta_{2 L} P_{2 L}(\cos \theta)\right],
$$

where $\sigma$ is the total cross section, $\beta_{2 L}$ the asymmetry parameters, and $P_{2 L}(\cos \theta)$ the Legendre polynomials. The total cross section $\sigma$ and asymmetry parameters $\beta$ for ionization of a $J_{i}$ level of the resonant or initial $(n=0)$ state can be written as (32)

$$
\sigma \propto \sum_{\substack{\ell m \\ M_{J_{i}}, M_{J_{+}}}} \rho M_{J_{i}} M_{J_{i}}\left|C_{\ell m}\left(M_{J_{i}} M_{J_{+}}\right)\right|^{2},
$$

and

$$
\begin{aligned}
\beta_{2 L}=\frac{4 L+1}{\sigma} \sum_{\substack{\ell \ell^{\prime} m \\
M_{J_{i}}, M_{J_{+}}}} & (-1)^{m}(2 \ell+1)\left(2 \ell^{\prime}+1\right) \rho M_{J_{i}} M_{J_{i}} C_{\ell m}\left(M_{J_{i}} M_{J_{+}}\right) \\
& \times C_{\ell^{\prime} m}^{*}\left(M_{J_{i}} M_{J_{+}}\right)\left(\begin{array}{ccc}
\ell & \ell^{\prime} & 2 L \\
m & -m & 0
\end{array}\right)\left(\begin{array}{ccc}
\ell & \ell^{\prime} & 2 L \\
0 & 0 & 0
\end{array}\right)
\end{aligned}
$$

where $\ell$ is an angular momentum component of the photoelectron and $m$ its projection in the laboratory frame. In Equation 2, $\left|C_{\ell m}\left(M_{J_{i}} M_{J_{+}}\right)\right|^{2}$ is the probability for photoionization of the $M_{J_{i}}$ level of the resonant or initial state leading to the $M_{J_{+}}$level of the ion.

In Equations 2 and 3, $\rho_{M_{J} M_{J}}$ is the population of a specific $M_{J_{i}}$ level in the optically aligned resonant state. For single-photon ionization processes $(n=0) \rho_{M_{s_{i}} M_{J_{1}}}$ is the population of the unaligned $M_{J_{0}}$ levels of the initial state. In the case of REMPI, we limit our discussion to the $n=2$ case, i.e. $\left(2+1^{\prime}\right)$ REMPI processes. For the low intensities of interest here, $\rho_{M_{J_{t}} M_{J_{t}}}$ for two-photon excitation has the general form 


$$
\rho_{M_{J_{i}} M_{J_{i}}}=\mathscr{N}\left|\sum_{k}\left(\begin{array}{ccc}
J_{i} & 1 & J_{k} \\
-M_{J_{i}} & 0 & M_{J_{i}}
\end{array}\right)\left(\begin{array}{ccc}
J_{k} & 1 & J_{0} \\
-M_{J_{i}} & 0 & M_{J_{i}}
\end{array}\right) B_{k}\right|^{2},
$$

where $\mathscr{N}$ is a normalization constant, $J_{k}$ is the total angular momentum of a dipole-allowed virtual state $|k\rangle$, and $B_{k}$ is related to the rotational line strength (33). Evaluation of Equation 4 generally requires summation over all dipole-allowed virtual states $|k\rangle$. For rotational branches other than the $Q$ branch, Equation 4 can be further simplified to a product of a $3-j$ symbol and rotational line strength $B(34)$,

$$
\rho_{M_{J_{i}} M_{J_{i}}}=\mathcal{N}\left(\begin{array}{ccc}
J_{i} & 2 & J_{0} \\
-M_{J_{i}} & 0 & M_{J_{i}}
\end{array}\right)^{2}
$$

However, in the case of a $Q$ branch, evaluation of $\rho_{M_{J_{i}} M_{J_{i}}}$ requires two factors, $B_{0}$ and $B_{2}$,

$$
\rho_{M_{J_{i} M_{J_{i}}}}=\mathcal{N}\left|\left(\begin{array}{ccc}
J_{i} & 2 & J_{0} \\
-M_{J_{i}} & 0 & M_{J_{i}}
\end{array}\right) B_{2}+B_{0}\right|^{2}
$$

Note that $B_{0}$ contains no polarization information but is crucial in determining the alignment $\rho_{M_{J_{i}} M_{J_{i}}}$ of the intermediate state for $\left(2+1^{\prime}\right)$ REMPI via the $Q$ rotational branch.

By choosing a coupling scheme intermediate between Hund's cases (a) and (b) for the resonant and ionic states and making use of multipletspecific final-state wave functions, $C_{\ell m}\left(M_{J_{t}} M_{J_{+}}\right)$has the form (32)

$$
\begin{aligned}
& C_{\ell m}\left(M_{J_{i}} M_{J_{+}}\right)=(\pi / 3)^{1 / 2}\left[\left(2 J_{+}+1\right)\left(2 J_{i}+1\right)\left(2 S_{i}+1\right)\right]^{1 / 2} \\
& \times \sum(-1)^{M_{J_{i}}+S_{+}-\Omega_{i}+\Sigma_{i}+1 / 2}\left(2 J_{t}+1\right)\left(2 J_{r}+1\right) C_{n_{+}^{\prime} n_{+}} C_{n^{\prime} n} \\
& \times\left[1+(-1)^{\mathrm{P}}\right]\left(\begin{array}{ccc}
J_{+} & J_{i} & J_{t} \\
-M_{J_{+}} & M_{J_{i}} & m_{t}
\end{array}\right)\left(\begin{array}{ccc}
S_{e} & \ell & J_{r} \\
-m_{e} & -m & m_{r}
\end{array}\right) \\
& \times\left(\begin{array}{ccc}
J_{r} & 1 & J_{t} \\
-m_{r} & \mu_{0} & -m_{t}
\end{array}\right)\left[\sum \tilde{I}_{\ell \lambda_{\mu} ; \Sigma_{e}}\left(\begin{array}{ccc}
J_{r} & 1 & J_{t} \\
-\lambda_{r} & \mu & -\lambda_{t}
\end{array}\right)\right. \\
& \times\left(\begin{array}{ccc}
S_{e} & \ell & J_{r} \\
-\Sigma_{e} & -\lambda & \lambda_{r}
\end{array}\right)\left(\begin{array}{ccc}
J_{+} & J_{i} & J_{t} \\
-\Omega_{+} & \Omega_{i} & \lambda_{t}
\end{array}\right)\left(\begin{array}{ccc}
S_{+} & S_{e} & S_{i} \\
\Sigma_{+} & \Sigma_{e} & -\Sigma_{i}
\end{array}\right)
\end{aligned}
$$




$$
\begin{aligned}
& +\sum \tilde{I}_{\ell \mu ; \Sigma_{e}}(-1)^{p_{i}+q_{t}}\left(\begin{array}{ccc}
J_{r} & 1 & J_{t} \\
-\lambda_{r} & \mu & -\lambda_{t}
\end{array}\right)\left(\begin{array}{ccc}
S_{e} & \ell & J_{r} \\
-\Sigma_{e} & -\lambda & \lambda_{r}
\end{array}\right) \\
& \left.\times\left(\begin{array}{ccc}
J_{+} & J_{i} & J_{t} \\
-\Omega_{+} & -\Omega_{i} & \lambda_{t}
\end{array}\right)\left(\begin{array}{ccc}
S_{+} & S_{e} & S_{i} \\
\Sigma_{+} & \Sigma_{e} & \Sigma_{i}
\end{array}\right)\right],
\end{aligned}
$$

with

$$
\begin{aligned}
& \tilde{I}_{\ell \lambda \mu ; \Sigma_{e}}=\sum_{\Lambda_{f} \Sigma_{f}}\left\langle\Lambda_{+} \lambda \mid \Lambda_{f}\right\rangle\left\langle\Sigma_{+} \Sigma_{e} \mid \Sigma_{f}\right\rangle I_{\ell \lambda \mu}\left(\Lambda_{f} \Sigma_{f}\right), \\
& I_{\ell \lambda \mu}=(-i)^{\ell} e^{i \eta_{e}} \int d R \chi_{v_{+}}^{*}(R) r_{f i}^{\ell \lambda \mu}(R) \chi_{v_{i}}(R),
\end{aligned}
$$

and

$$
P=\Delta J+\Delta S+2 S_{e}+\Delta p+\Delta q+\ell+1,
$$

where $\Delta J=J_{+}-J_{i}, \Delta S=S_{+}-S_{i}, \Delta p=p_{+}-p_{i}, \Delta q=q_{+}-q_{i} ; p$ and $q$ are parity indices (35); $J_{r}$ and $J_{t}$ are angular momentum transfers; $\left|\Lambda_{f} \Sigma_{f}\right\rangle$ are the multiplet-specific final-state wave functions; $\mathrm{C}_{n_{+}^{\prime} n_{+}}$and $\mathrm{C}_{n^{\prime} n}$ are expansion coefficients for the wave functions of the rovibronic states of the ion and resonant states (31), respectively; and the summations in Equation 7 go over all possible indices. In Equation $9, r_{f i}^{\ell \lambda \mu}(R)$ is the photoelectron matrix element, and $I_{\ell \lambda \mu}$ is the vibrationally averaged photoelectron matrix element between the resonant state and the photoelectron continuum wave function.

PHOTOELECTRON MATRIX ELEMENT A central quantity in these studies is the matrix element for photoejection of an electron from a bound molecular orbital $\phi_{i}$ into a photoelectron continuum orbital $\Psi_{f, \mathbf{k}}^{(-)}(\mathbf{r})$. Here $\mathbf{k}$ is the momentum of the photoelectron and (-) denotes incoming-wave boundary conditions. For linear molecules, the partial wave components $\psi_{k \ell m}^{(-)}$of $\Psi_{f, \mathbf{k}}^{(-)}(\mathbf{r})$ are defined by an expansion in spherical harmonics about $\hat{\mathbf{k}}$ of the photoelectron

$$
\Psi_{f, \mathbf{k}}^{(-)}(\mathbf{r})=\left(\frac{2}{\pi}\right)^{1 / 2} \sum_{\ell m} i^{\ell} \psi_{k \ell m}^{(-)}(\mathbf{r}) Y_{\ell m}^{*}(\hat{\mathbf{k}}) .
$$

Single-center expansions of $\psi_{k \ell_{m}}^{(-)}(\mathbf{r})$ and $\phi_{i}\left(\mathbf{r}^{\prime}\right)$, e.g.

$$
\psi_{k \ell m}^{(-)}=\sum_{\ell^{\prime} \lambda} g_{\ell, \ell^{\prime} \lambda}(k, r) \mathscr{D}_{m \lambda}^{\ell} Y_{\ell^{\prime} \lambda}\left(\hat{\mathbf{r}}^{\prime}\right)
$$

define partial wave photoelectron matrix elements $r_{f i}^{\rho \lambda \mu}(R)$ in the molecular frame for ionization of orbital $\phi_{i}\left(\mathbf{r}^{\prime}\right)$, i.e. 


$$
r_{f i}^{\ell \lambda \mu}(R)=\sum_{\ell^{\prime}, \ell_{0}}\left\langle g_{\ell, \ell^{\prime} \lambda}(k, r, R) Y_{\ell^{\prime} \lambda}\left(\hat{\mathbf{r}}^{\prime}\right)\left|r Y_{1 \mu}\left(\hat{\mathbf{r}}^{\prime}\right)\right| \phi_{i \ell_{0}}(r, R) Y_{\ell_{0}, \lambda_{0}}\left(\hat{\mathbf{r}}^{\prime}\right)\right\rangle
$$

where $R$ denotes a dependence on internuclear distance, $\mu$ is the light polarization index in the molecular frame, $\mathscr{D}_{m \lambda}^{\ell}$ is the rotational matrix in Edmonds' notation (36), $\lambda$ is the projection of $\ell$ in the molecular frame, and $\psi_{k \ell m}$ is a partial wave component of the photoelectron orbital (31) with momentum $\mathbf{k}$.

Equation 13 reveals an important underlying dynamical aspect of molecular photoelectron wave functions. Whereas only $\ell=\ell^{\prime}$ terms are allowed in Equation 13 for atomic systems with their central fields, where the angular momentum of the photoelectron must be conserved, $\ell \neq \ell^{\prime}$ terms arise in Equation 13 owing to the nonspherical potential fields of molecular ions. This angular momentum coupling between partial waves $\ell$ and $\ell^{\prime}$ is brought about by the torques associated with the molecular ion potential and makes a molecular photoelectron orbital an admixture of angular momentum components. The use of molecular photoelectron orbitals that correctly incorporate such angular momentum coupling is essential in studying rotationally resolved photoelectron dynamics.

PARITY SELECTION RULES Parity selection rules $(32,37-39)$ govern changes of rotational angular momentum upon ionization. Equations 7 and 10 yield the parity selection rule

$$
\Delta J+\Delta S+\Delta p+\Delta q+\ell=e v e n
$$

for an intermediate coupling scheme between Hund's cases (a) and (b). This parity selection rule is the same as that of Dixit \& McKoy (37) and of Xie \& Zare (38). In Hund's case (b) limit, Equation 14 becomes (32, 37-39)

$$
\Delta N+\Delta p+\ell=o d d
$$

with $\Delta N=N_{+}-N_{\mathrm{i}}$. For a $\Sigma-\Sigma$ transition, Equation 15 reduces to $\Delta N+\ell=$ odd $(40)$.

\section{Ionization of Asymmetric Tops}

ROTATIONALLY RESOLVED PHOTOELECTRON SPECTRA The formulation for rotationally resolved photoelectron spectra of asymmetric tops is basically the same as that for linear molecules. The coefficients $C_{\ell m}\left(M_{J_{i}} M_{J_{+}}\right)$of Equations 2 and 3 for asymmetric tops with $C_{2 \mathrm{~V}}$ molecular symmetry have the form (41) 


$$
\begin{aligned}
& C_{\ell m}\left(M_{J_{i}} M_{J_{+}}\right)=\sqrt{\frac{4 \pi}{3}} \frac{1}{2}\left[\left(2 J_{i}+1\right)\left(2 J_{+}+1\right)\left(2 N_{i}+1\right)\left(2 N_{+}+1\right)\left(2 S_{i}+1\right)\right]^{1 / 2} \\
& \times \sum(-1)^{Q}\left(2 N_{t}+1\right)\left(\begin{array}{ccc}
S_{+} & \frac{1}{2} & S_{i} \\
M_{S_{+}} & m_{\sigma} & -M_{S_{i}}
\end{array}\right)\left(\begin{array}{ccc}
N_{+} & S_{+} & J_{+} \\
M_{N_{+}} & M_{S_{+}} & -M_{J_{+}}
\end{array}\right) \\
& \times\left(\begin{array}{ccc}
N_{i} & S_{i} & J_{i} \\
M_{N_{i}} & M_{S_{i}} & -M_{J_{i}}
\end{array}\right)\left(\begin{array}{ccc}
N_{+} & N_{i} & N_{t} \\
-M_{N_{+}} & M_{N_{i}} & m_{i}
\end{array}\right)\left(\begin{array}{ccc}
N_{t} & 1 & \ell \\
-m_{t} & \mu_{0} & m
\end{array}\right) \\
& \times a_{N_{i} \zeta_{i} K_{i}} a_{N_{+} \zeta_{+} K_{+}} \tilde{I}_{h \ell \lambda \mu}^{\tau \tau}\left(\Lambda_{f} \Sigma_{f}\right) b_{h \ell \lambda}^{\gamma \tau}\left(\Lambda_{f} \Sigma_{f}\right) \\
& \times\left[1+(-1)^{\Delta p+\Delta N+\ell+1}\right]\left[\left(\begin{array}{ccc}
N_{+} & N_{i} & N_{t} \\
-K_{+} & K_{i} & K_{t}
\end{array}\right)\left(\begin{array}{ccc}
N_{t} & 1 & \ell \\
-K_{t} & \mu & \lambda
\end{array}\right)\right. \\
& \left.+(-1)^{p_{+}}\left(\begin{array}{ccc}
N_{+} & N_{i} & N_{t} \\
K_{+} & K_{i} & K_{t}
\end{array}\right)\left(\begin{array}{ccc}
N_{t} & 1 & \ell \\
-K_{t} & \mu & \lambda
\end{array}\right)\right] \\
& Q=\Delta N+\Delta M_{J}-S_{i}+M_{S_{i}}-\mu_{0}-m+M_{N_{+}}+K_{i}-1 / 2, \\
& \tilde{I}_{h \ell \lambda_{\mu}}^{\tau}\left(\Lambda_{f} \Sigma_{f}\right)=\left\langle\gamma_{+} \tau_{+} \gamma_{e} \tau_{e} \mid \Lambda_{f}\right\rangle\left\langle M_{S_{+}} m_{o} \mid \Sigma_{f}\right\rangle I_{h e \lambda \mu}^{\psi \tau}\left(\Lambda_{f} \Sigma_{f}\right),
\end{aligned}
$$

and

$$
\begin{aligned}
I_{h \ell \lambda \mu}^{\gamma \tau}\left(\Lambda_{f} \Sigma_{f}\right) & =\sqrt{\frac{2}{\pi}} \frac{(-i)^{\ell}}{k} \\
& \times \int d R d r \psi_{+}^{*}(\mathbf{r}, R) \chi_{v_{+}}^{*}(R) \psi_{h \ell \lambda}^{*(-) \gamma \tau}(\mathbf{r}, R) r Y_{1 \mu} \psi_{i}(\mathbf{r}, R) \chi_{v_{i}}(R),
\end{aligned}
$$

where $\gamma$ is one of the irreducible representations (IR) of the molecular point group; $\tau$ is a component of this representation; $h$ distinguishes between different bases for the same IR corresponding to the same value of $\ell ; \zeta=K_{a}-K_{c} ; K_{a}$ and $K_{c}$ are the projections of the total angular momentum along the $a$ and $c$ axes, respectively; $b_{h \in \lambda}^{\gamma \tau}$ are the expansion coefficients; and the $a_{N \zeta K}$ coefficients are determined by diagonalizing the rigid rotor Hamiltonian in a basis of symmetric top eigenfunctions.

PHOTOELECTRON MATRIX ELEMENT For asymmetric top molecules, the partial wave components $\psi_{k h \ell m}^{(-) y^{\prime}}$ of $\Psi_{f, \mathbf{k}}^{(-)}(\mathbf{r})$ are similarly defined by an expansion in generalized harmonics about $\hat{\mathbf{k}}$ of the photoelectron

$$
\Psi_{f, \mathbf{k}}^{(-)}(\mathbf{r})=\left(\frac{2}{\pi}\right)^{1 / 2} \sum_{\ell m} i^{\ell} \psi_{k h \ell m}^{(-) \gamma \tau}(\mathbf{r}) X_{h \ell m}^{* \gamma \tau}(\hat{\mathbf{k}})
$$


With single-center expansions for $\psi_{k h m_{m}}^{(-) \gamma^{\tau}}(\mathbf{r})$ and $\phi_{i}^{\gamma^{t^{\prime}}}\left(\mathbf{r}^{\prime}\right)$, partial wave photoelectron matrix elements $r_{h e \lambda_{\mu}}^{\gamma \tau}(R)$ in the molecular frame for ionization out of orbital $\phi_{i}^{\gamma^{\prime} \tau^{\prime}}\left(\mathbf{r}^{\prime}\right)$ are given by (41)

$$
r_{h \ell \lambda \mu}^{\gamma \tau}(R)=\sum_{\ell^{\prime}, \ell_{0}}\left\langle g_{h l, \ell^{\prime} \lambda}^{\gamma \tau}(k, r, R) X_{h \ell^{\prime} \lambda}^{\gamma^{\tau} \lambda}\left(\hat{\mathbf{r}}^{\prime}\right)\left|r Y_{1 \mu}\left(\hat{\mathbf{r}}^{\prime}\right)\right| \phi_{i h^{\prime} \ell_{0}}^{\gamma^{\prime} \tau^{\prime}}(r, R) X X_{h 0_{0}, \lambda_{0}}^{\gamma^{\prime} \tau^{\prime}}\left(\hat{\mathbf{r}}^{\prime}\right)\right\rangle
$$

Note that while $\ell \neq \ell^{\prime}$ terms arise in both Equations 13 and 21, owing to the nonspherical potential fields of molecular ions, for nonlinear molecules, coupling of the projections of angular momenta along appropriate axes can become pervasive.

PARITY SELECTION RULES Here we choose a left-handed coordinate system for the molecular internal $x, y$, and $z$ axes with the molecular $z$ axis as the symmetry axis. With the symmetry properties of the asymmetric top (41), it can be shown that $\Delta K_{\eta}$ is even (odd) when $\Delta N+\Delta p$ is even (odd). Here $\eta$ is the principal axis lying along the molecular $x$ axis, and the $K_{\eta}$ is the projection of the total angular momentum along this axis. Thus we have the selection rule (41)

$$
\Delta K_{\eta}+\ell=o d d .
$$

The selection rules also depend on which principal axis coincides with the molecular $z$ axis. With the properties of 3-j symbols, Equation 16 provides additional selection rules (42):

$$
\mu+\lambda= \begin{cases}\Delta K_{a} & \text { if } a / / z / / \text { the symmetry axis. } \\ \Delta K_{b} & \text { if } b / / z / / \text { the symmetry axis. } \\ \Delta K_{c} & \text { if } c / / z / / \text { the symmetry axis. }\end{cases}
$$

To relate the selection rules of Equations 22 and 23, a relationship among $\Delta K_{a}, \Delta K_{b}$, and $\Delta K_{c}$ is essential. This relationship, which can be obtained from the symmetry properties of an asymmetric top (42), is

$$
\Delta K_{a}+\Delta K_{c}=\operatorname{even}(\text { odd }) \leftrightarrow \Delta K_{b}=\text { even }(o d d) .
$$

Determination of $\mu+\lambda$ now becomes a critical step in application of these selection rules. Whereas Equations $22-24$ are suitable for any nonlinear polyatomic molecule, $\mu+\lambda$ must be determined specifically for a given molecular symmetry.

\section{Ionization of Symmetric Tops}

The $C_{\ell m}\left(M_{J_{i}} M_{J_{+}}\right)$coefficients of Equations 2 and 3 for symmetric tops such as $\mathrm{CH}_{3}$ have the form (43) 


$$
\begin{aligned}
& C_{\ell m}\left(M_{J_{i}} M_{J_{+}}\right)=\sqrt{\frac{4 \pi}{3}}\left[\left(2 J_{i}+1\right)\left(2 J_{+}+1\right)\left(2 N_{i}+1\right)\left(2 N_{+}+1\right)\left(2 S_{i}+1\right)\right]^{1 / 2} \\
& \times \sum(-1)^{Q}\left(2 N_{t}+1\right)\left(\begin{array}{ccc}
S_{+} & \frac{1}{2} & S_{i} \\
M_{S_{+}} & m_{\sigma} & -M_{S_{i}}
\end{array}\right)\left(\begin{array}{ccc}
N_{+} & S_{+} & J_{+} \\
M_{N_{+}} & M_{S_{+}} & -M_{J_{+}}
\end{array}\right) \\
& \times\left(\begin{array}{ccc}
N_{i} & S_{i} & J_{i} \\
M_{N_{i}} & M_{S_{i}} & -M_{J_{i}}
\end{array}\right)\left(\begin{array}{cccc}
N_{+} & N_{i} & N_{t} \\
-M_{N_{+}} & M_{N_{i}} & m_{t}
\end{array}\right)\left(\begin{array}{ccc}
N_{t} & 1 & \ell \\
-m_{t} & \mu_{0} & m
\end{array}\right) \\
& \times \tilde{I}_{h \ell \lambda \mu}^{\tau}\left(\Lambda_{f} \Sigma_{f}\right) b_{h \ell \lambda}^{\gamma \tau}\left(\Lambda_{f} \Sigma_{f}\right)\left[\left(\begin{array}{cccc}
N_{+} & N_{i} & N_{t} \\
-K_{+} & K_{i} & K_{t}
\end{array}\right)\left(\begin{array}{ccc}
N_{t} & 1 & \ell \\
-K_{t} & \mu & \lambda
\end{array}\right)\right. \\
& +(-1)^{p_{i}}\left(\begin{array}{cccc}
N_{+} & N_{i} & N_{t} \\
-K_{+} & -K_{i} & K_{t}
\end{array}\right)\left(\begin{array}{ccc}
N_{t} & 1 & \ell \\
-K_{t} & \mu & \lambda
\end{array}\right) \\
& +(-1)^{p_{+}}\left(\begin{array}{lll}
N_{+} & N_{i} & N_{t} \\
K_{+} & K_{i} & K_{t}
\end{array}\right)\left(\begin{array}{ccc}
N_{t} & 1 & \ell \\
-K_{t} & \mu & \lambda
\end{array}\right) \\
& \left.+(-1)^{p_{i}+p_{+}}\left(\begin{array}{lll}
N_{+} & N_{i} & N_{t} \\
K_{+} & -K_{i} & K_{t}
\end{array}\right)\left(\begin{array}{ccc}
N_{t} & 1 & \ell \\
-K_{t} & \mu & \lambda
\end{array}\right)\right] .
\end{aligned}
$$

Note that no parity selection rules relating $\Delta p, \Delta N$, and $\ell$ are obtained for photoionization of symmetric tops, since the partial wave components of each electronic continuum have different phase factors (43). However, Equation 25 does reveal that $\mu+\lambda= \pm \Delta K$ and $\mu+\lambda= \pm\left(K_{+}+K_{i}\right)$.

\section{Computational Procedures}

In applications of our procedure to REMPI of Rydberg states, we use the improved virtual orbital (IVO) method (44) to obtain the wave function of the resonant state. For single-photon ionization, we assume a selfconsistent-field wave function for the ground state of the molecule. For the final state we assume a frozen-core Hartree-Fock model in which the photoelectron orbital is obtained as a solution of a one-electron Schrödinger equation containing the Hartree-Fock potential of the molecular ion.

To obtain the photoelectron orbital, we have used an iterative procedure, based on the Schwinger variational principle (31), to solve the Lippmann-Schwinger equation. This procedure begins by approximating the static-exchange potential of the ionic core by a separable potential of the form 


$$
\mathbf{U}\left(\mathbf{r}, \mathbf{r}^{\prime}\right)=\sum_{i j}\left\langle\mathbf{r}|\mathbf{U}| \alpha_{i}\right\rangle\left(\mathbf{U}^{-1}\right)_{i j}\left\langle\alpha_{j}|\mathbf{U}| \mathbf{r}^{\prime}\right\rangle
$$

where the matrix $\mathbf{U}^{-1}$ is the inverse of the matrix with the elements $(U)_{i j}=\left\langle\alpha_{i}|\mathbf{U}| \alpha_{j}\right\rangle$ and the $\alpha$ 's are discrete basis functions such as Cartesian or spherical Gaussian functions. $\mathbf{U}$ is twice the static-exchange potential with the long-term Coulomb potential removed. The Lippmann-Schwinger equation with this separable potential $\mathbf{U}\left(\mathbf{r}, \mathbf{r}^{\prime}\right)$ can be readily solved and provides an approximate photoelectron orbital $\phi_{k}^{(0)}$. These $\phi_{k}^{(0)}$ can be iteratively improved to yield converged solutions to the Lippmann-Schwinger equation containing the full static-exchange potential. A few iterations of this variational method provide highly converged solutions for the molecular photoelectron orbitals.

\section{RESULTS AND DISCUSSION}

\section{(2+1) REMPI of Linear Molecules ( $\mathrm{HBr}$ and $\mathrm{OH}$ )}

As a first example, in Figure 1 we compare our calculated ion rotational distributions for $\mathrm{HBr}^{+}$in its $X^{2} \Pi_{1 / 2}$ ground state for $(2+1)$ REMPI via the $S(2)$ branch of the $F^{1} \Delta_{2}(5 p \pi)$ Rydberg state (45) with the experimental data of Xie \& Zare (19). Our studies of these spectra were motivated by measurements of the populations of the individual parity components of each ion rotational level by laser-induced fluorescence (19). These spectra show a strongly ( - ) parity-favored ion rotational distribution for photoionization of the $(+)$ parity component of the $J_{i}=4$ level of the $5 p \pi$ Rydberg orbital of the $F^{1} \Delta_{2}$ state, which has about $97 \% p$ character. These (-) parity-favored ion distributions can be readily understood on the basis of the parity selection rules of Equation 14 and the dominance of the photoelectron matrix element by its $l=0$ and $l=2$ components for photoionization of this $5 p \pi$ orbital. However, the most striking result is that in addition to the dominant population seen in the (-) parity components of the $\Lambda$ doublet of the ion rotational levels, about $20 \%$ population is observed in the $(+)$ parity components that cannot be accounted for on the basis of atomiclike behavior. Earlier theoretical studies of these ion rotational distributions had predicted extremely small populations for the (+) parity levels (46). Autoionization was subsequently proposed as a possible underlying mechanism responsible for the population observed in the $(+)$ parity components of these ion rotational levels (46).

Figure 1 shows our calculated spectra (45) for photoionization of the optically aligned $J_{i}=4$ level [via the $S(2)$ branch], and an unaligned $J_{i}=4$ level, along with the measured spectra (19). The agreement between the calculated and measured ion distributions is excellent. Note that, on the 

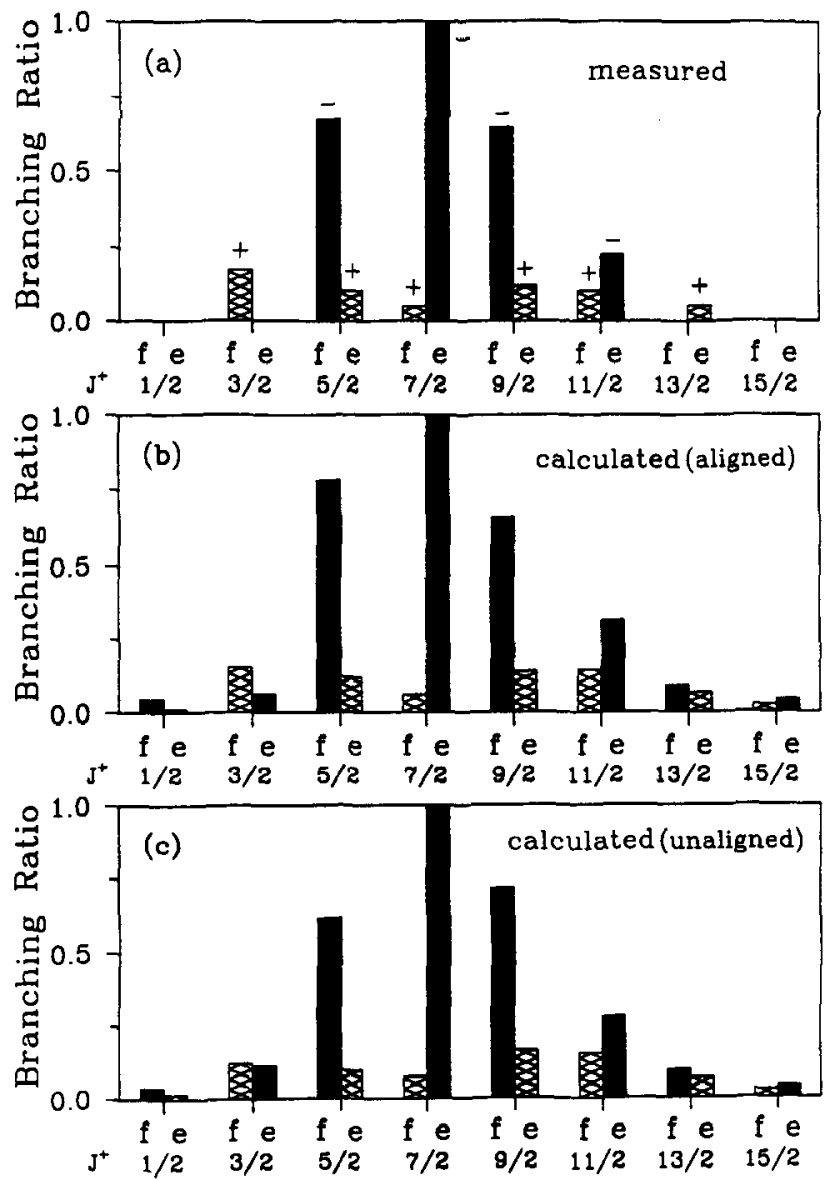

Figure 1 The measured and calculated rotational distributions of $\mathrm{HBr}^{+}$for $(2+1)$ REMPI via the $S(2)$ branch of the $F^{\prime} \Delta_{2}$ state. The calculated distributions in $b$ and $c$ are for an aligned and unaligned resonant state, respectively, and for a photoelectron energy of $2.33 \mathrm{eV}$. " +" and " - " indicate the parity of each rotational level.

basis of selection rules, the $20 \%$ population seen in the $(+)$ parity component (cross-hatched bars) of the $\Lambda$ doublet is due to odd partial wave components of the photoelectron matrix elements that arise from angular momentum coupling in the molecular photoelectron orbitals. The present results clearly demonstrate that the population seen in the $(+)$ levels arises quite naturally in a correct quantitative description of direct photoionization and that an autoionization mechanism need not be invoked (46). Examination of the photoelectron matrix element $r_{f i}^{\ell \lambda \mu}$ of Equation 13 reveals that the $f$ wave of the $5 p \pi \rightarrow k \delta$ ionization channel makes the 
dominant contribution to the population of these $(+)$ parity levels of the ion. Such behavior is entirely nonatomiclike (45). Comparison of the ion distributions for the unaligned $J_{i}=4$ level with those for an optically aligned level and with the measured spectra serves to illustrate that, although not large, the effect of alignment can be important.

Figure 2 shows the measured and calculated rotationally resolved photoelectron spectra for $(2+1)$ REMPI of OH via the $O_{11}(11)$ branch of the
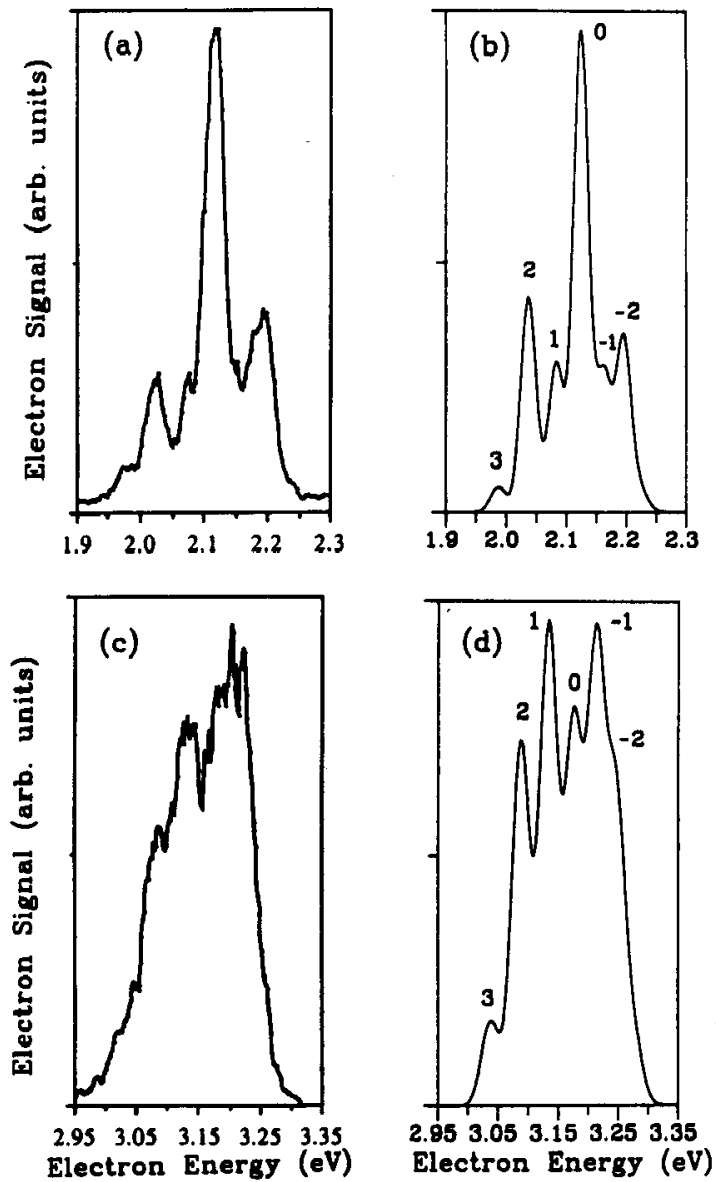

Figure 2 Measured and calculated rotationally resolved photoelectron spectra for $(2+1)$ REMPI of OH: (a) measured spectrum for the $D^{2} \Sigma^{-}(3 p \sigma)$ state, $v_{i}=0 \rightarrow v_{+}=0, O_{11}(11)$ rotational branch; $(b)$ calculated $D^{2} \Sigma^{-}$photoelectron spectrum, assuming a Gaussian line shape with an FWHM of $30 \mathrm{meV} ;(c)$ measured spectrum for the $3^{2} \Sigma^{-}(4 s \sigma)$ state, $v_{i}=0 \rightarrow$ $v_{+}=0, O_{11}(11)$ rotational branch; $(d)$ calculated $3^{2} \Sigma^{-}$photoelectron spectrum, assuming a Gaussian line shape with an FWHM of $35 \mathrm{meV}$. The labeling of peaks in the calculated spectra indicates the change of rotational quantum number $\Delta N=N^{+}-N_{i}$. 
$D^{2} \Sigma^{-}(3 p \sigma)$ (Figures $2 a$ and $b$ ) and $3^{2} \Sigma^{-}(4 s \sigma)$ (Figures $2 c$ and $d$ ) Rydberg states (27). For ionization via the $D^{2} \Sigma^{-}$state, strong $\Delta N=$ even signals were observed in the photoelectron spectrum, in contrast to the $\Delta N=$ odd distribution expected on the basis of ionization of the $3 p \sigma(34.9 \% s$ and $63.3 \% p$ character) Rydberg orbital into even $(\ell=0,2)$ components of the photoelectron continua. On the other hand, the rotationally resolved photoelectron spectra for the $3^{2} \Sigma^{-}$Rydberg state of Figures $2 c$ and $d$ reveal a qualitatively different and much broader distribution with prominent $\Delta N=$ even and $\Delta N=$ odd transitions. The appearance of these spectra arises from greater $\ell$ mixing in this higher Rydberg orbital $(54.3 \% s$ and $42.7 \% p$ character).

To illustrate the rich photoionization dynamics of these strong even $\Delta N$ peaks in the spectra, Figure 3 shows the magnitude of the (incoming-wave normalized) partial-wave amplitude $\left|D_{t}^{(-)}\right|$as a function of photoelectron kinetic energy for the $(a) 3 p \sigma \rightarrow k \sigma$ and $(b) 3 p \sigma \rightarrow k \pi$ channels for photoionization of the $3 p \sigma$ orbital of the $D^{2} \Sigma^{-}$Rydberg state of $\mathrm{OH}$. Two Cooper minima, which are due to sign changes in the $\ell=2$ components of the photoelectron matrix elements around the minimum in $\left|D_{\ell}^{(-)}\right|$, are clearly seen at a kinetic energy of about $3.0 \mathrm{eV}$ in the $k \sigma$ and $k \pi$ channels. The actual sign changes in the matrix elements occur in the principal-value (standing-wave normalized) dipole amplitude $D_{\ell}^{P}$, as shown in the insets of Figure 3 for the $\ell=2$ wave. The energy positions of both minima in $\left|D_{\ell}^{(-)}\right|$differ somewhat from those of the Cooper zeros in $D_{\ell}^{P}$. These shifts reflect the influence of angular momentum coupling in the electronic continua on the transformation relating $D_{\ell}^{P}$ to $D_{\ell}^{(-)}$. The strong even $\Delta N=0$ peaks of Figures $2 a$ and $b$ can, in fact, be shown to result from the presence of Cooper minima in the $\ell=2$ wave of the photoelectron channels. Depletion of the $d$ wave $(\ell=2)$ contribution to the photoelectron matrix element in the vicinity of both Cooper minima subsequently enhances the relative importance of the odd $\ell$ waves and, hence, that of the even $\Delta N$ rotational peaks. Similar effects of Cooper minima on rotationally resolved photoelectron spectra have also been observed in REMPI of the $f^{1} \Pi(3 p \sigma)$ state of NH (11) and the $D^{2} \Sigma^{+}(3 p \sigma)$ state of NO (47).

\section{Single-Photon Ionization of Linear Molecules ( $N O$ and $\mathrm{CO}$ )}

Figure 4 shows the (a) measured and $(b)$ calculated ZEKE-PFI photoelectron spectra for single-photon ionization of rotationally cold NO $\left(X^{2} \Pi_{1 / 2}, v^{\prime \prime}=0\right)$ molecules leading to $\mathrm{NO}^{+}\left(X^{1} \Sigma^{+}, v_{+}=1\right)$ by coherent VUV radiation (48). The calculated ion rotational distributions assume a temperature of $5 \mathrm{~K}$. These spectra were calculated for a photoelectron energy of $50 \mathrm{meV}$ and convoluted with a Gaussian detection function with a full-width at half-maximum (FWHM) of $2 \mathrm{~cm}^{-1}$. In Figure $4 b$ each 

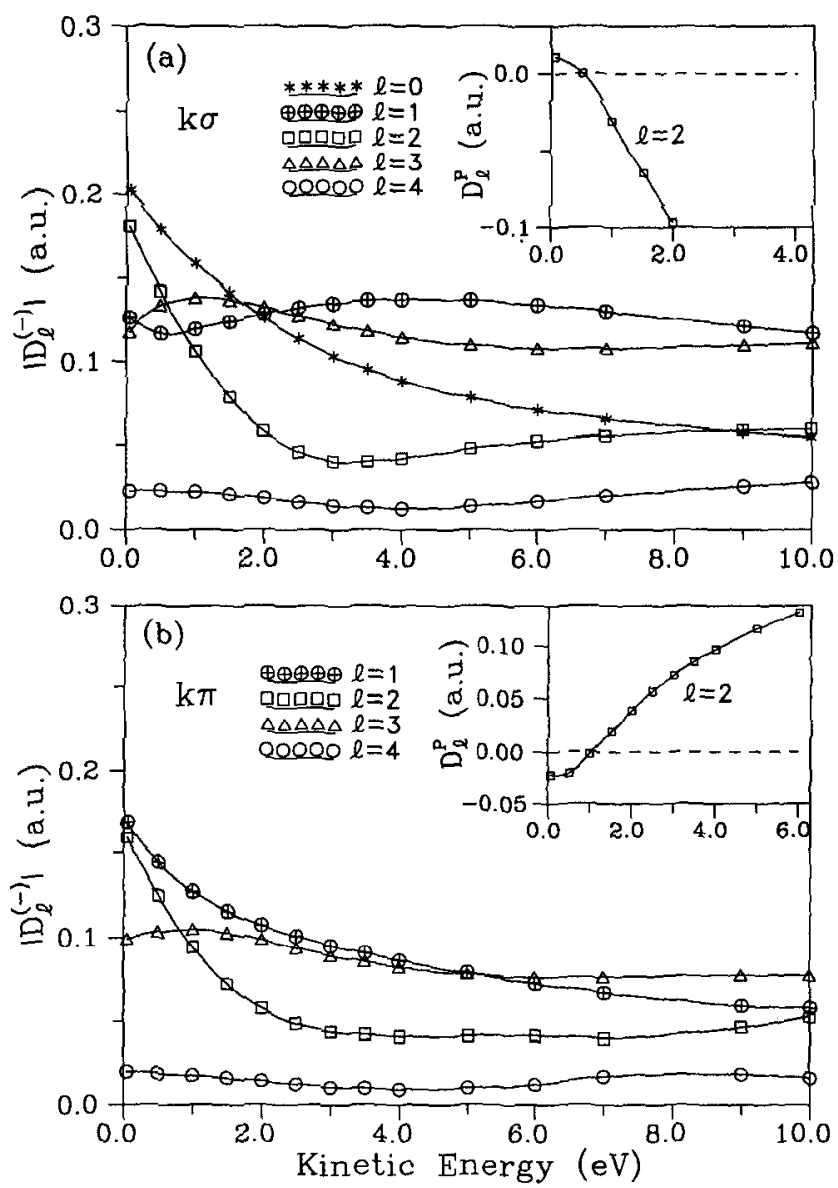

Figure 3 Magnitude of the partial-wave photoionization matrix elements as a function of kinetic energy for the $(a) 3 p \sigma \rightarrow k \sigma$ and $(b) 3 p \sigma \rightarrow k \pi$ channels for the $D^{2} \Sigma^{-}(3 p \sigma)$ state of $\mathrm{OH}$. The inset shows the principal-value dipole amplitude $D_{\ell}^{P}$ for the $\ell=2$ component.

branch is associated with a letter designation that refers only to the change in angular momentum apart from spin. The label $1^{\prime}$ denotes the $N^{\prime \prime}=1$, $J^{\prime \prime}=1 / 2$ level. The agreement between these measured and calculated ZEKE spectra is excellent.

In Figure 4, only small changes in total angular momentum are observed $(|\Delta J| \leqslant 5 / 2)$ with branch intensities that fall off rapidly as $|\Delta J|$ increases. The observation of branches with $|\Delta J| \leqslant 5 / 2$ suggests that the dominant contributions to the photoelectron matrix element at threshold are from $\ell \leqslant 1$. This differs from expectations based on the calculated magnitudes 


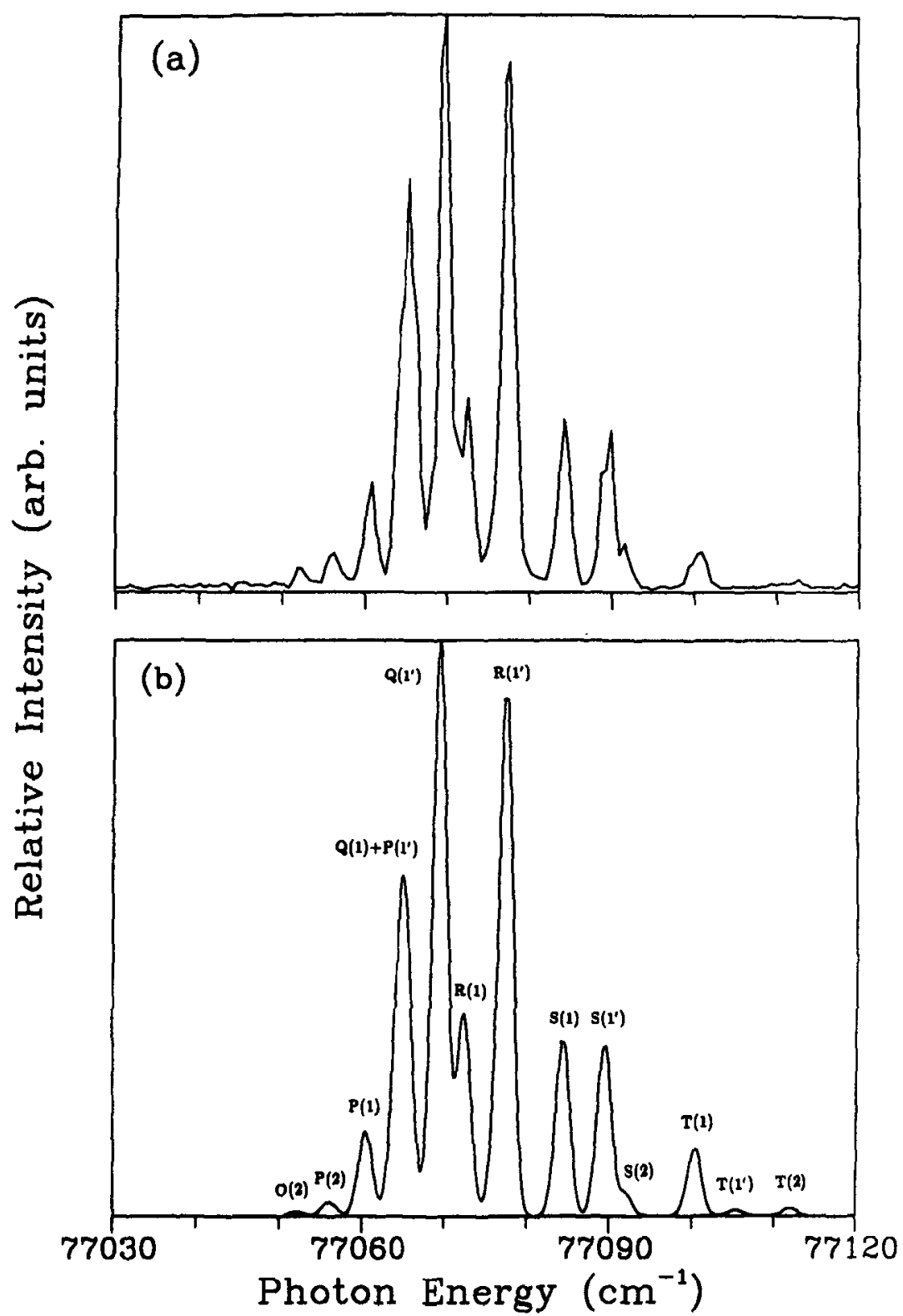

Figure 4 The (a) measured and $(b)$ calculated ZEKE-PFI photoelectron spectra for singlephoton ionization of rotationally cold NO $\left(X^{2} \Pi_{1 / 2}\right)$ by coherent VUV radiation. 
of these partial wave dipole elements where the $d$ and $f$ components are seen to be substantial (48). Unusually strong $s$ and $d$ waves are also predicted in addition to the $p$ and $f$ partial waves expected in photoionization of the $2 \pi$ orbital with its $84 \% d\left(\ell_{0}=2\right)$ character (48). This behavior reflects the significant coupling of angular momentum in the photoelectron continuum due to torques associated with the molecular ion potential. Given that these calculated photoelectron matrix elements yield an accurate representation of the measured threshold photoelectron spectra, one may conclude that photoexcitation into high- $\ell$ partial waves is not accompanied by observable changes in ion-core angular momentum of comparable magnitude. That such large changes in angular momentum $\Delta J(\Delta N)$ are not seen in these spectra in spite of the significant magnitudes of the photoelectron matrix element for higher $\ell(=3)$ is probably due to interference between these partial waves in the photoelectron continua. The effect of such interference on ion rotational distributions has also been seen in $(2+1)$ REMPI via the $f^{1} \Pi(3 p \sigma)$ and the $g^{1} \Delta(3 p \pi)$ Rydberg states of NH (11) and in (2+1) REMPI via the ${ }^{2} \Phi(3 d \pi)$ Rydberg state of $\mathrm{SH}(13)$.

In Figures 5 and 6, we show the calculated and measured rotationally resolved ZEKE-PFI photoelectron spectra for photoionization of the $1 \pi$ orbital of the $X^{1} \Sigma^{+}\left(v^{\prime \prime}=0\right)$ state of CO leading to the $v^{+}=1$ level of the $A^{2} \Pi_{1 / 2}$ and $A^{2} \Pi_{3 / 2}$ ions, respectively, for a temperature of $50 \mathrm{~K}(49,50)$. The calculated spectra assumed a photoelectron kinetic energy of $50 \mathrm{meV}$ and were convoluted with a Gaussian detector function with an FWHM of $2 \mathrm{~cm}^{-1}$. Because these spectra were calculated with the same dynamical matrix elements for both spin-orbit components of the ion, differences in these ion distributions must arise from the spin-orbit interaction, i.e. the projection of total electronic angular momentum on the internuclear axis $\Omega_{+}$in Equation 7. Agreement between the calculated and measured spectra is quite reasonable. Both calculated and measured spectra show $\Delta J=1 / 2$ to be the most intense peak and display transitions arising from higher rotational levels.

Parity selection rules of Equation 14 govern angular momentum transfer between the photoelectron and ion core upon ionization. Because the $\Lambda$ doublet (e/f) of the $A^{2} \Pi$ excited state rotational level is not resolved, it is impossible to assign a specific parity (even or odd $\ell$ ) to individual rotational transitions. Both parities can contribute to each rotational transition. Since the $1 \pi$ orbital has almost pure odd $(81 \% p$ and $11 \% f)$ character (49), dominant even ( $s$ and $d$ ) partial wave components would be expected for the photoelectrons on the basis of atomiclike propensity rule. The calculated photoelectron matrix elements show that this is indeed the case for photoionization of the $1 \pi$ orbital. 

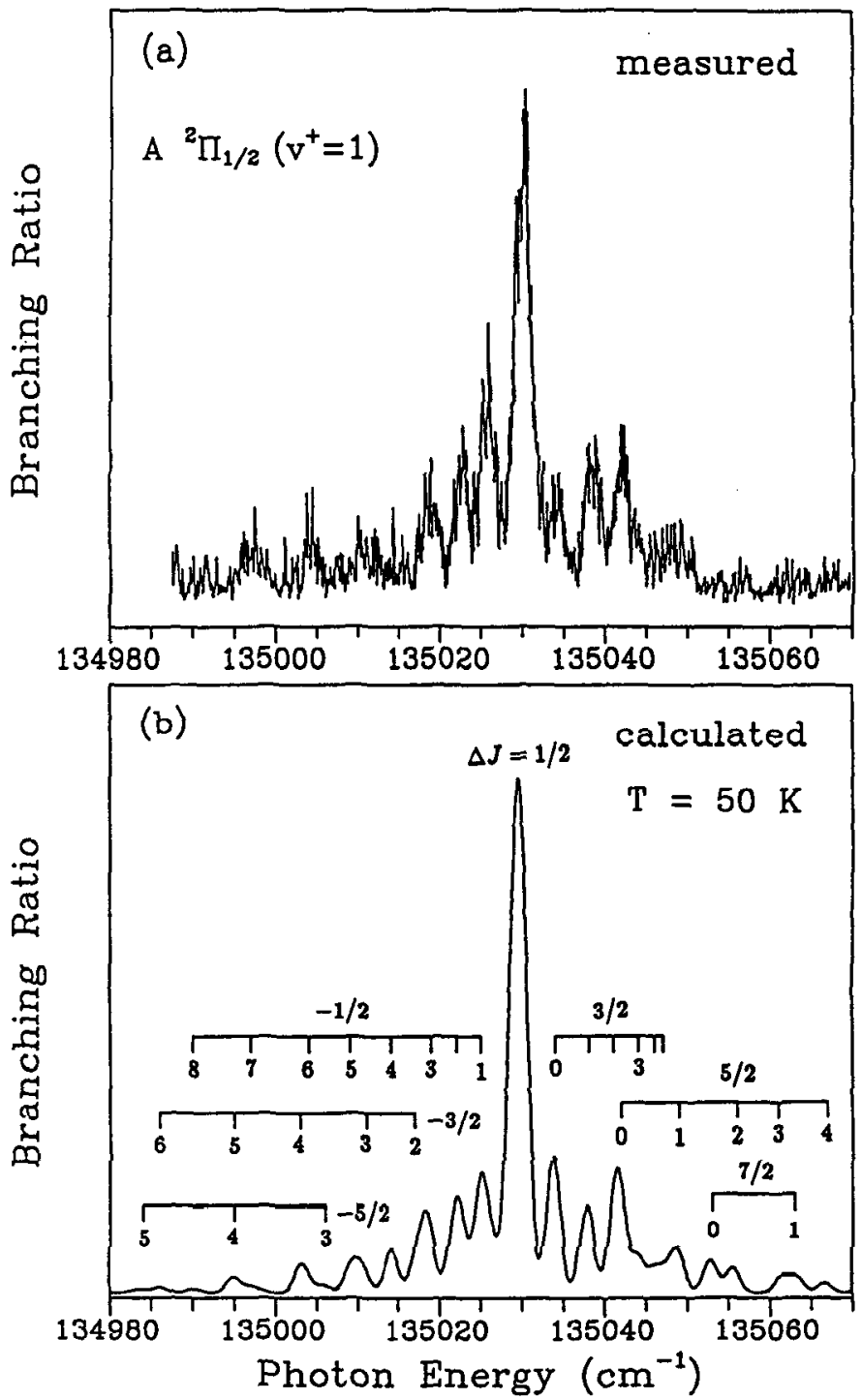

Figure 5 The $(a)$ measured and $(b)$ calculated ZEKE-PFI photoelectron spectra for ionization of the $1 \pi$ orbital of the $X^{1} \Sigma^{+}\left(v^{\prime \prime}=0\right)$ ground state of $C O$ leading to the $A^{2} \Pi_{1 / 2}$ $\left(v^{+}=1\right)$ spin-orbit component of $\mathrm{CO}^{+}$.

Figures 5 and 6 also show that the measured negative $\Delta J$ transitions are somewhat more intense than those of the calculated spectra, while the positive $\Delta J$ transitions have similar intensities for both the calculated and 

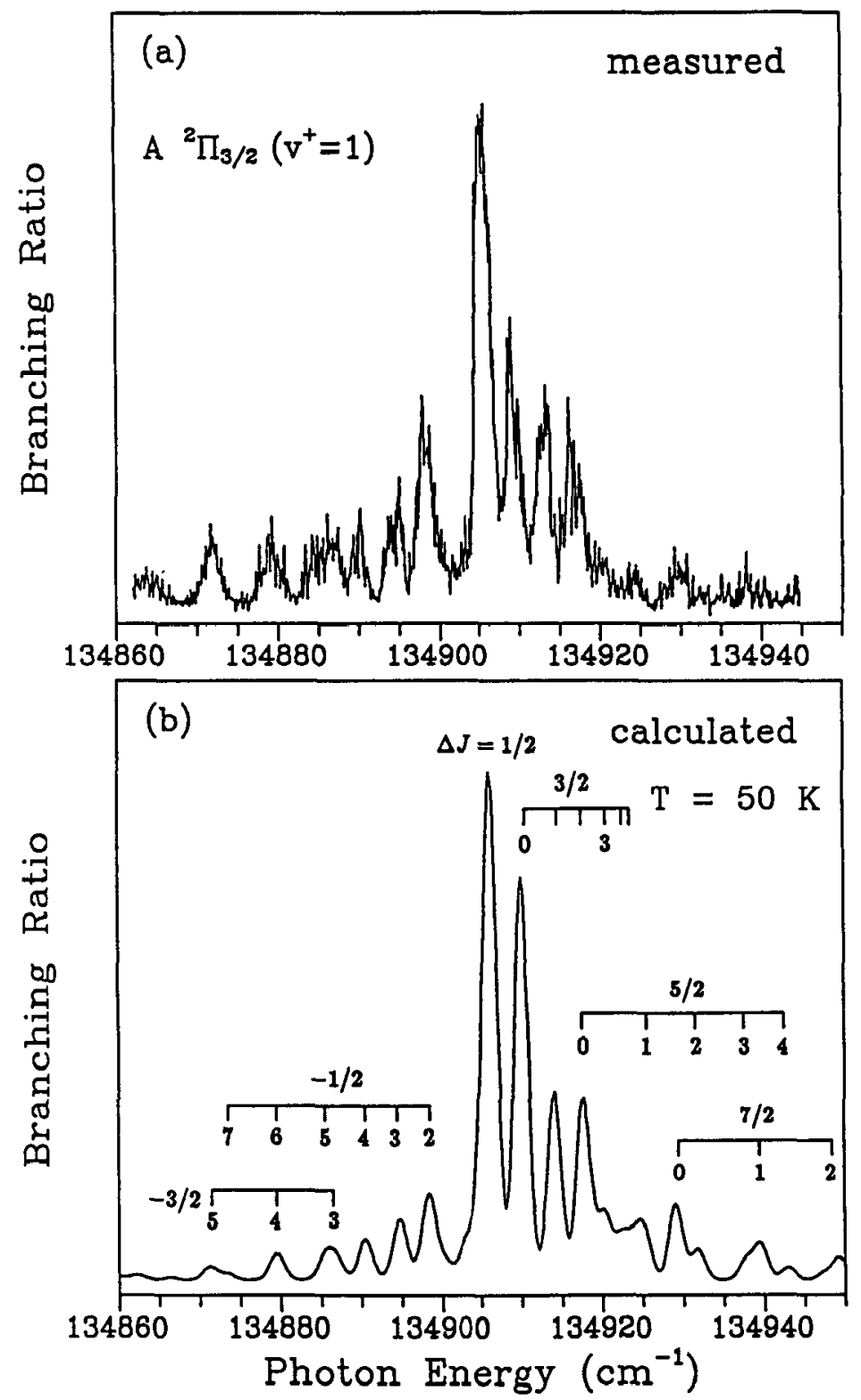

Figure 6 The $(a)$ measured and $(b)$ calculated ZEKE-PFI photoelectron spectra for ionization of the $1 \pi$ orbital of the $X^{1} \Sigma^{+}\left(v^{\prime \prime}=0\right)$ ground state of CO leading to the $A^{2} \Pi_{3 / 2}$ $\left(v^{+}=1\right)$ spin-orbit component of $\mathrm{CO}^{+}$. 


\section{WANG \& McKOY}

measured spectra. This behavior is quite common in rotational intensity profiles of PFI spectra for a wide range of systems. Field-induced autoionization has been used to explain such anomalous spectral profiles (22). This mechanism involves the interaction of high- $n$ Rydberg states $(\geqslant 150)$ with nearly degenerate low- $n$ Rydberg states converging to higher cation rovibronic ionization threshold. Application of the pulsed electric field lowers the ionization potential by an amount equal to the Stark shift and allows the low- $n$ Rydberg states to autoionize (18, 22, 51-54).

Single-Photon Ionization of Nonlinear Molecules $\left(\mathrm{H}_{2} \mathrm{O}\right.$, $\mathrm{H}_{2} \mathrm{CO}$, and $\mathrm{CH}_{3}$ )

Figure $7 a$ shows the rotationally resolved ZEKE-PFI spectrum of $\mathrm{H}_{2} \mathrm{O}$ for single-photon ionization of the $1 b_{1}$ orbital by coherent VUV radiation (55). This spectrum can be assigned to two types of rotational transitions, corresponding to specific changes in $K_{a}$ and $K_{c}$. Most of the strong spectral
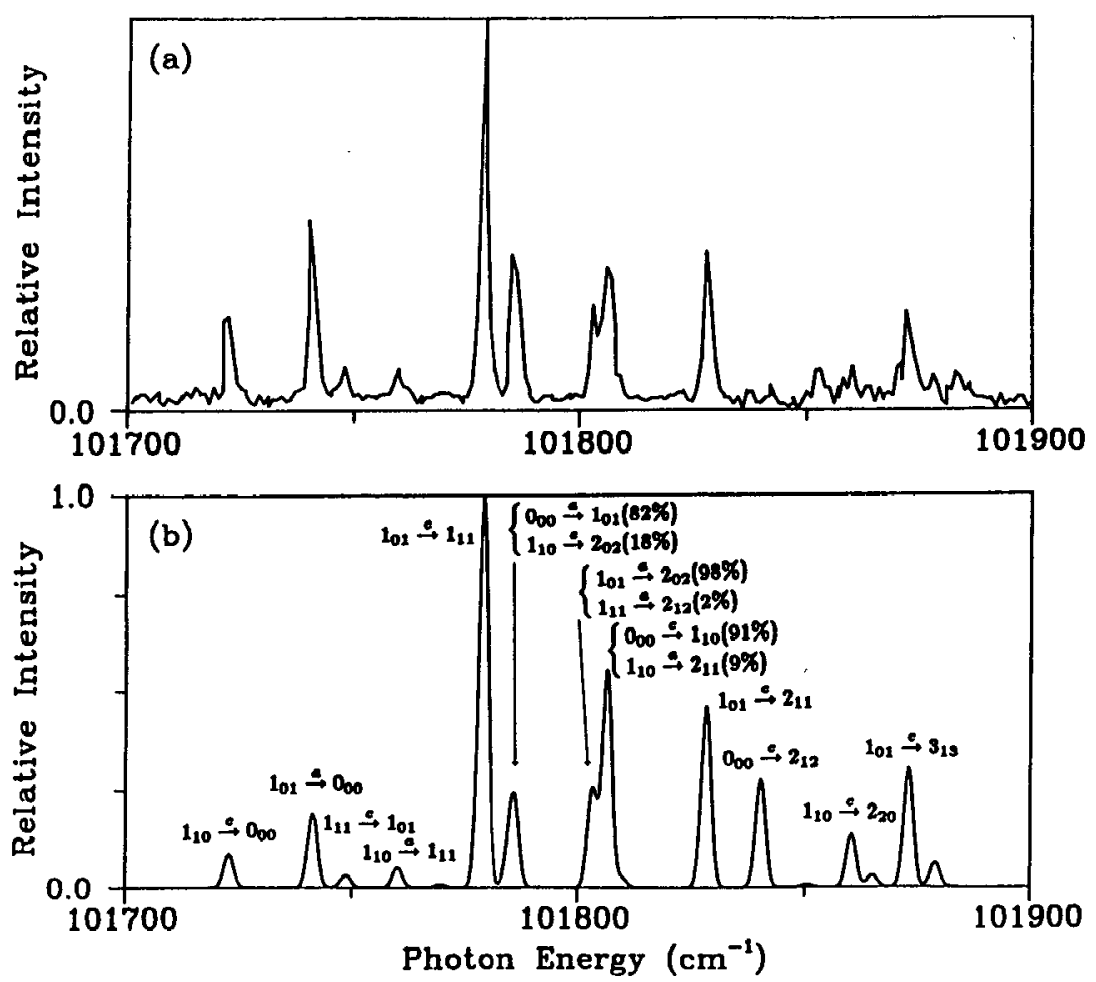

Figure 7 The $(a)$ measured and $(b)$ calculated ion rotational distributions for single-photon ionization of the $1 b_{1}$ orbital of the $\tilde{X}^{1} A^{1}$ ground state of jet-cooled $\mathrm{H}_{2} \mathrm{O}$. The $a$ and $c$ labels indicate type $a$ and type $c$ transitions, respectively. 
lines could be classified as type $c$ rotational transitions $\left(\Delta K_{a}=o d d\right.$, $\left.\Delta K_{c}=e v e n\right)$, but type $a$ transitions $\left(\Delta K_{a}=e v e n, \Delta K_{c}=o d d\right)$ are also clearly evident. Figure $7 b$ shows the calculated ion rotational distributions $(56,57)$ for photoionization of the $1 b_{1}$ orbital of the $\tilde{X}^{\prime} A_{1}(000)$ ground state of jet-cooled $\mathrm{H}_{2} \mathrm{O}$ leading to the $\tilde{X}^{2} B_{1}(000)$ ground state of the ion. A photoelectron kinetic energy of $50 \mathrm{meV}$ and a rotational temperature of $15 \mathrm{~K}$ are assumed in these calculations. Furthermore, we assume that there is no spin exchange during the jet-cooled expansion of room-temperature water. The calculated spectrum is convoluted with a Gaussian detection function with an FWHM of $1.5 \mathrm{~cm}^{-1}$. Agreement between the calculated and measured spectra is clearly encouraging, except for the $0_{00} \rightarrow 2_{12}$ transition for which the calculated intensity is somewhat stronger than its measured value.

The underlying dynamics of these photoelectron spectra is quite rich. Here we assume that the molecular $z$ axis coincides with the $C_{2}$ symmetry axis and that the $x$ axis lies in the plane of the molecule (ion). The molecular $x, y$, and $z$ axes hence coincide with the $a, c$, and $b$ axes, respectively. For photoionization of the $1 b_{1}$ orbital of $\mathrm{H}_{2} \mathrm{O}$, from Equation 24 we have

$$
\Delta K_{a}+\ell=o d d
$$

and

$$
\Delta K_{a}+\Delta K_{c}=o d d .
$$

Clearly, both type $a$ and type $c$ transitions are allowed, and type $b\left[\Delta K_{a}=\right.$ even $($ odd $)$ and $\Delta K_{c}=$ even $($ odd $\left.)\right]$ transitions are forbidden. Furthermore, Equation 27 shows that type $a$ transitions $\left(1_{01} \rightarrow 0_{00}, 0_{00} \rightarrow 1_{01}, 1_{01} \rightarrow 2_{02}\right.$, and $1_{11} \rightarrow 2_{12}$ ) arise from odd (almost pure $p$ ) wave contributions to the photoelectron matrix element of Equation 21. These $p$ waves of the $k a_{1}$ and $k b_{1}$ continua are entirely molecular in origin, since the almost pure $p$ $(99.7 \%)$ character of the $1 b_{1}$ orbital would lead only to $s$ and $d$ (even) photoelectron continua in an atomiclike picture. The strong type $c$ transitions in these spectra arise from $s$ and $d$ (even) wave components of the photoelectron matrix elements. The $k a_{2}$ continuum makes almost no contribution to these type $a$ transitions, owing to the negligible $f$ (odd) wave. In a recent study using multichannel quantum defect theory (MQDT), Child \& Jungen (30) predicted that only type $c$ transitions are allowed for photoionization of the $1 b_{1}$ orbital of ground state water. Gilbert \& Child (58) further proposed a rotational autoionization mechanism, based on polarization-induced quasiautoionizing state-mixing between the $1 b_{1} \rightarrow n d$ and $1 b_{1} \rightarrow n p$ Rydberg series, in an effort to account for these type $a$ transitions. In contrast, the above analysis $(56,57)$ shows that these type $a$ transitions arise quite naturally in a correct quantitative 


\section{WANG \& McKOY}

description of the direct molecular photoionization process itself and a polarization-induced autoionization mechanism need not be invoked.

Figure 8 shows the $(a)$ measured and $(b)$ calculated rotationally resolved ZEKE-PFI spectra of $\mathrm{H}_{2} \mathrm{CO}$ for single-photon ionization of the nonbonding $2 b_{2}$ orbital by coherent VUV radiation (43). The calculated spectrum assumes a rotational temperature of $7 \mathrm{~K}$ and a photoelectron kinetic energy of $50 \mathrm{meV}$ and is convoluted with a Gaussian detection function with an FWHM of $1.8 \mathrm{~cm}^{-1}$. Furthermore, we assume that no spin exchange occurs during the jet-cooled expansion of room-temperature formaldehyde, i.e. a population ratio of $2: 1$ for the ortho (B symmetry) to para (A symmetry) species. The agreement between calculated and measured spectra is very encouraging.

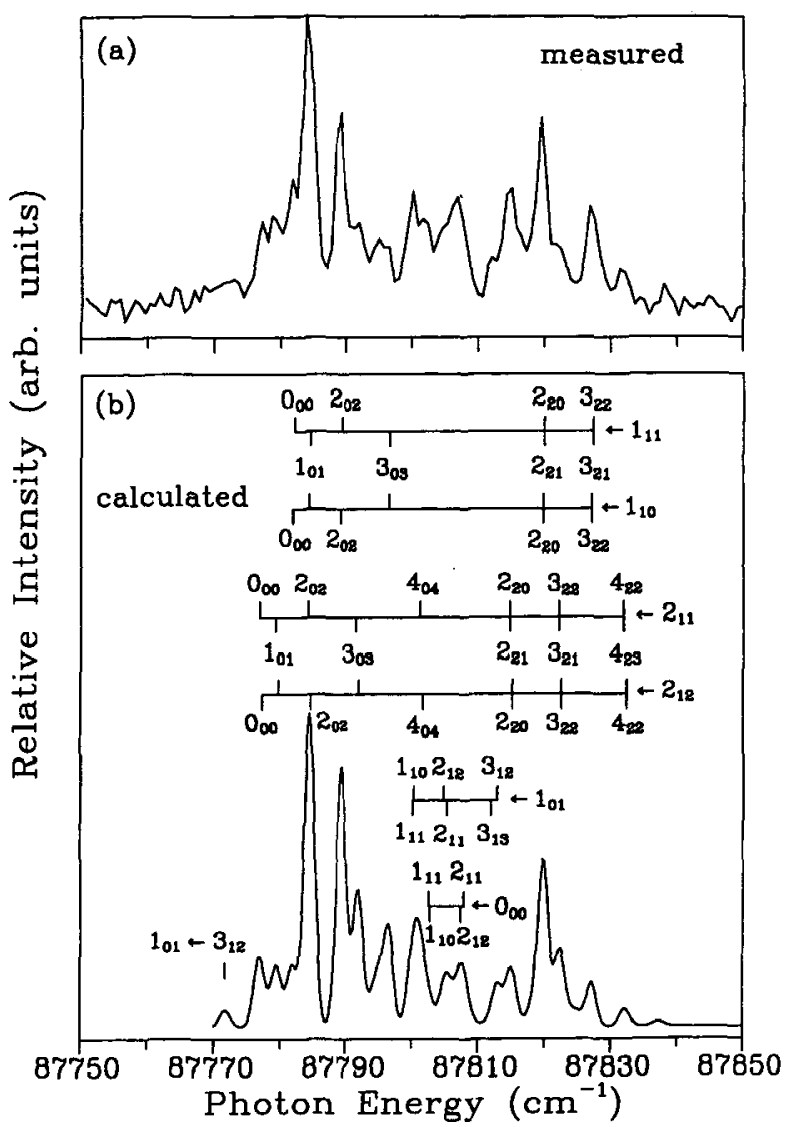

Figure 8 The $(a)$ measured and $(b)$ calculated ion rotational distributions for single-photon ionization of the $2 b_{2}$ orbital of the $\tilde{X}^{\prime} A_{1}$ ground state of jet-cooled $\mathrm{H}_{2} \mathrm{CO}$. 
In our calculations we assume that the molecular $z$ axis coincides with the $C_{2}$ symmetry axis and that the $x$ axis lies in the plane of the formaldehyde molecule (ion). The molecular $x, y$, and $z$ axes hence coincide with the $b, c$, and $a$ axes, respectively. For photoionization of the $2 b_{2}$ orbital of $\mathrm{H}_{2} \mathrm{CO}$, from Equation 24 we have

$$
\Delta K_{\mathrm{b}}+\ell=o d d
$$

and

$$
\Delta K_{b}+\Delta K_{c}=o d d .
$$

Clearly, both type $c\left(\Delta K_{a}=\right.$ odd and $\Delta K_{c}=$ even $)$ and type $b\left(\Delta K_{a}=\right.$ odd and $\left.\Delta K_{c}=o d d\right)$ transitions are allowed, and type $a\left(\Delta K_{a}=e v e n\right.$ and $\left.\Delta K_{c}=o d d\right)$ and other type $b\left(\Delta K_{a}=e v e n\right.$ and $\left.\Delta K_{c}=e v e n\right)$ transitions are forbidden. Furthermore, Equations 29 and 30 show that the allowed type $b$ transitions arise from odd partial wave contributions to the photoelectron matrix elements of Equation 21 whereas the type $c$ transitions arise from even angular momentum components of these matrix elements.

In Figure $8 b$, we label several of the more important transitions out of rotational levels of the ground state of the neutral species leading to different rotational levels of the ion. The quantum numbers used as labels are $N_{K_{a} K_{c}}$. Note that in this figure we use the same set of quantum numbers to designate type $c$ transitions out of the $1_{11}$ level and type $b$ transitions out of the $1_{10}$ level, since the energies of these transitions are essentially equal. A similar labeling is also adopted for transitions out of the $2_{11}$ and $2{ }_{12}$ rotational levels. Because the moments of inertia $I_{b}$ and $I_{c}$ are almost the same for $\mathrm{H}_{2} \mathrm{CO}\left(\mathrm{H}_{2} \mathrm{CO}^{+}\right)$, the rotationally resolved ZEKE-PFI spectra are very congested. Unlike the spectra for $\mathrm{H}_{2} \mathrm{O}$, type $b$ transitions, which arise from odd waves, cannot be distinguished from type $c$ transitions, which arise from even waves.

Figure 9 shows the calculated threshold photoelectron spectra for the individual type $c$ (Figure $9 a$ ) and type $b$ (Figure $9 b$ ) transitions of Figure 8 for single-photon ionization of the $2 b_{2}$ orbital of the ground state of $\mathrm{H}_{2} \mathrm{CO}$. Furthermore, our calculated spectra show a total intensity for type $b$ transitions about twice as large as that for type $c$ transitions. This large intensity of the type $c$ transitions cannot be accounted for on the basis of atomiclike propensity rules because the $2 b_{2}$ orbital of formaldehyde has $4.8 \% p, 80.0 \% d, 3.7 \% f, 9.1 \% g\left(\ell_{0}=4\right)$, and $1.5 \% h\left(\ell_{0}=5\right)$ character. The strong even-wave character of the $2 b_{2}$ orbital would be expected to lead to dominant odd partial wave contributions to the photoelectron matrix elements and, hence, to dominant type $b$ transitions. This unexpected contribution from even partial waves of the photoelectron matrix element is quite molecular in origin and arises from strong $\ell$-mixing caused 


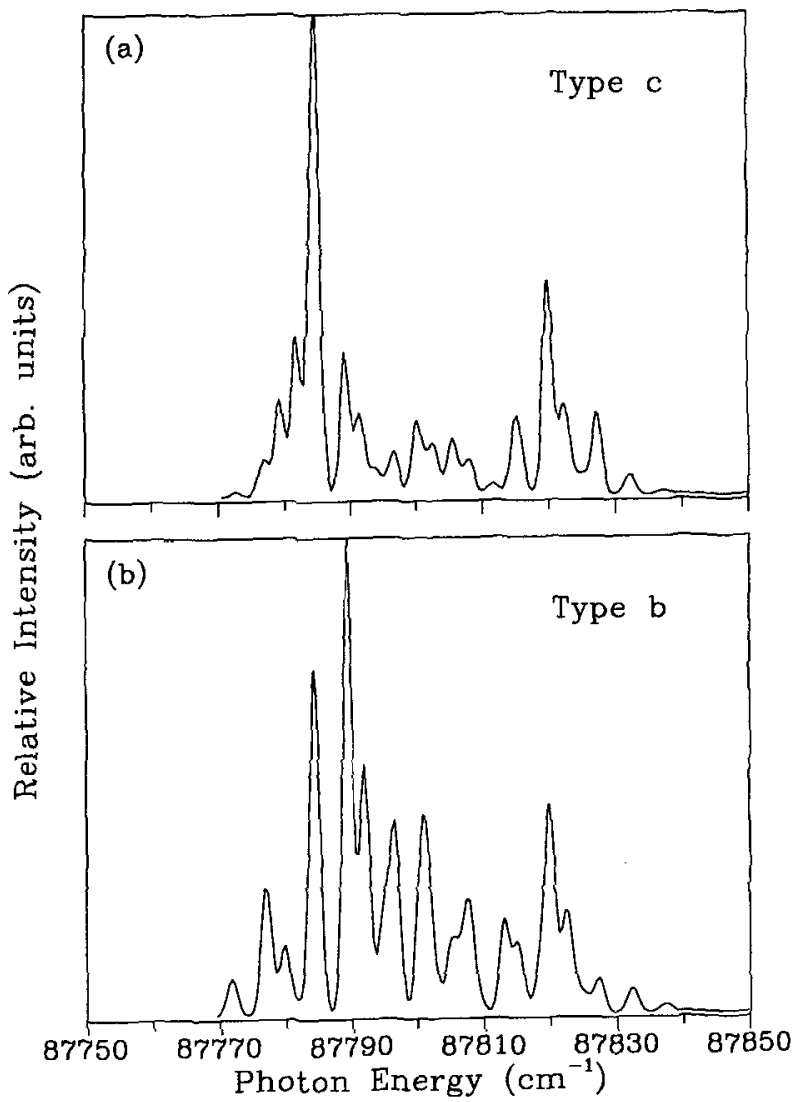

Figure 9 Calculated photoelectron spectra for the (a) type $c$ and $(b)$ type $b$ transitions of Figure $8 b$ for single-photon ionization of the $2 b_{2}$ orbital of the $\tilde{X}^{1} A_{1}$ ground state of jetcooled $\mathbf{H}_{2} \mathbf{C O}$. The type $b$ to type $c$ intensity ratio is about 1.9 .

by the nonspherical molecular ion potentials. Such comparisons between measured and calculated spectra can be helpful in unravelling severely congested photoelectron spectra.

Figure 10 shows the $(a)$ measured $(59)$ and $(b)$ calculated rotationally resolved ZEKE-PFI spectra of $\mathrm{CH}_{3}\left(\tilde{X}^{2} A_{2}^{\prime \prime}\right)$ for single-photon ionization of the $1 a_{2}^{\prime \prime}$ orbital by coherent VUV radiation leading to the $\tilde{X}^{1} A_{1}^{\prime}$ ground state of the ion (43). The calculated spectrum assumes a rotational temperature of $250 \mathrm{~K}$ and a photoelectron kinetic energy of $50 \mathrm{meV}$ and is convoluted with a Gaussian detection function with an FWHM of 2.5 $\mathrm{cm}^{-1} \cdot \mathrm{CH}_{3}$ and $\mathrm{CH}_{3}^{+}$are oblate symmetric tops, belonging to the $D_{3 h}$ point 


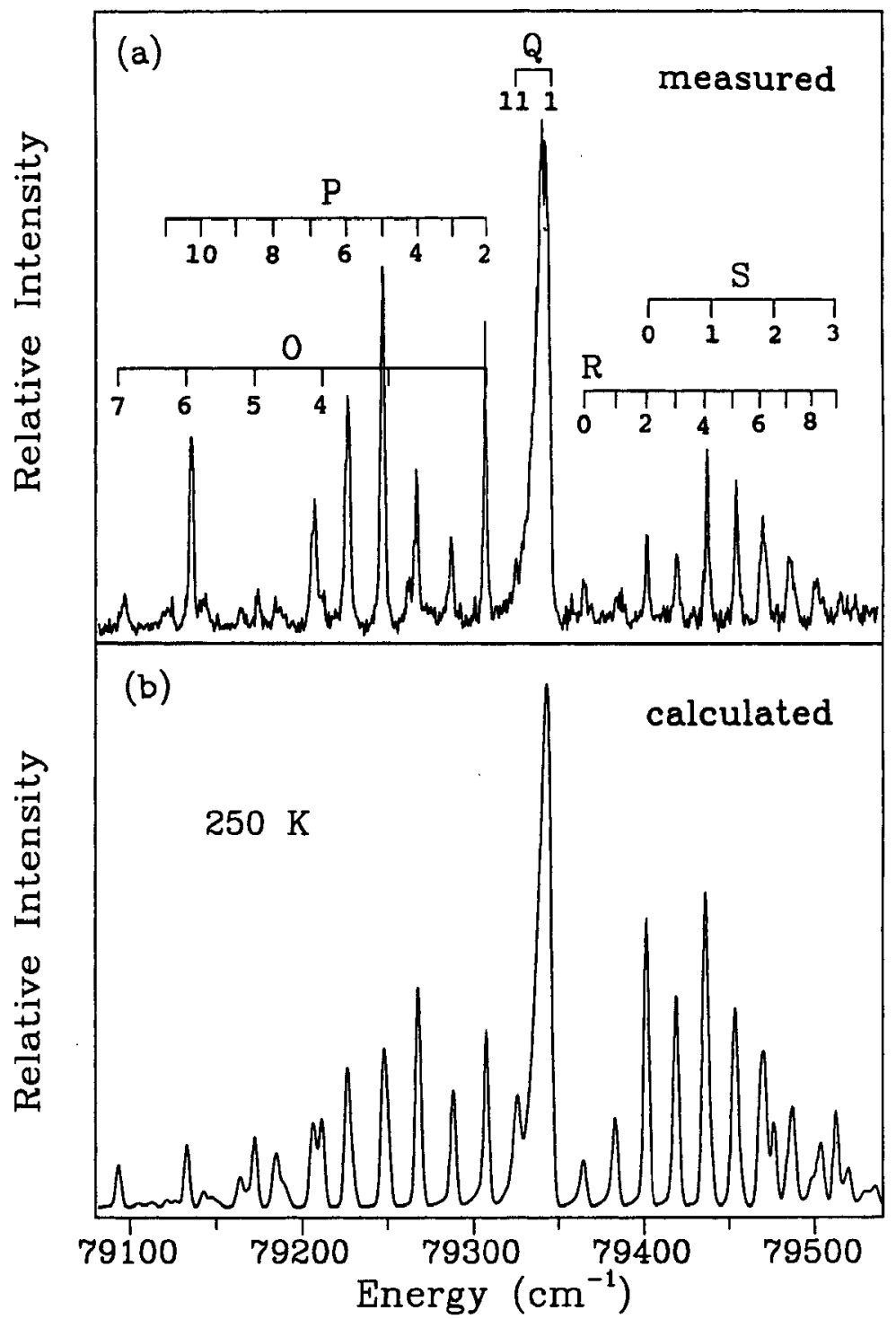

Figure 10 The (a) measured and (b) calculated ion rotational distributions for single-photon ionization of the $1 a_{2}^{\prime \prime}$ orbital of the $\tilde{X}^{2} A_{2}^{\prime \prime}$ ground state of jet-cooled $\mathrm{CH}_{3}$ leading to the $\tilde{X}^{1} A_{1}^{\prime}$ ground state of the ion.

proup. For the $D_{3 h}$ group, the total wave function of $\mathrm{CH}_{3}$ should have either $A_{2}^{\prime}$ or $A_{2}^{\prime \prime}$ symmetry in order to obey the Pauli principle. The wave functions for ortho and para species have $A_{1}^{\prime}(I=3 / 2)$ and $E^{\prime}(I=1 / 2)$ 
symmetries, respectively. Because $\Gamma_{\text {spin }} \otimes \Gamma_{\text {rot }} \supset \Gamma_{\text {total }}$, the rotational wave function must have $A_{2}^{\prime}, A_{2}^{\prime \prime}, E^{\prime}$, or $E^{\prime \prime}$ symmetry. Therefore, the rotational levels belonging to $A_{1}^{\prime}$ and $A_{1}^{\prime \prime}$ symmetries are not initially populated, i.e. levels with $J=$ even, $K=0$, and the $A_{1}(K=3 n)$ level of the $A_{1}-A_{2}$ pair are forbidden. Due to the spin statistical weight of $4: 2$ between ortho and para species, the $K=3 n$ levels $\left(A_{2}^{\prime}\right.$ or $A_{2}^{\prime \prime}$ ) have a statistical weight twice that of the $K=3 n+1$ levels ( $E^{\prime}$ or $E^{\prime \prime}$ ).

Agreement between the calculated and measured spectra is very encouraging in spite of some discrepancies that arise from rotational autoionization at negative $\Delta N$ transitions (22). Our calculations reveal that $\Delta K=0$ are the dominant transitions (up to $95 \%$ ) and that $\Delta K= \pm 2$ (not labeled) transitions are much weaker. Even though the $\Delta K=$ odd transitions are also dipole allowed, their intensities are very weak and they do not appear in the spectrum. Note that $\Delta K=$ odd transitions are associated with $\mu+\lambda=$ odd partial waves of the photoelectron, which, in turn, must originate from the $f\left(\ell_{0}=3\right)$ component of the $1 a_{2}^{\prime \prime}$ orbital of the ground state. Because the $1 a_{2}^{\prime \prime}$ orbital is essentially an out-of-plane $2 p$ orbital localized on the central carbon atom, its $f$ wave is negligible. This leads to a $\Delta K=$ even selection rule.

\section{$\mathrm{N}_{2}$ and $\mathrm{CO}$ Ion Distributions at High Photoelectron Energies}

In Figure 11, we show the measured and calculated ion rotational distributions of $\mathrm{N}_{2}^{+}\left(B^{2} \Sigma_{\mathrm{u}}^{+}\right)$and $\mathrm{CO}^{+}\left(B^{2} \Sigma^{+}\right)$resulting from single-photon ionization of the $2 \sigma_{\mathrm{u}}$ and $4 \sigma$ orbitals of the ground state of $\mathrm{N}_{2}$ and $\mathrm{CO}$, respectively $(20,60)$. The calculated spectra for $\mathrm{N}_{2}$ and $\mathrm{CO}$ assumed temperatures of 14 and $18 \mathrm{~K}$, respectively. The measured spectra were extracted from the $\mathrm{N}_{2}^{+}\left(B^{2} \Sigma_{\mathrm{u}}^{+} \rightarrow X^{2} \Sigma_{\mathrm{g}}^{+}\right)$and $\mathrm{CO}^{+}\left(B^{2} \Sigma^{+} \rightarrow X^{2} \Sigma^{+}\right)$fluorescence spectra (20). For $\mathrm{N}_{2}$, data were generated over the range $20 \leqslant h v_{\text {exc }} \leqslant 220 \mathrm{eV}$, but with its lower signal intensities, results were only obtained from $23 \leqslant h v_{\mathrm{exc}} \leqslant 145 \mathrm{eV}$ for $\mathrm{CO}$.

For $\mathrm{N}_{2}$, as the energy increases, population is transfered from low $N^{+}$ levels to higher ones, owing to the increasing propensities for larger $\Delta N$ transitions. For the weakly populated $N^{+}=5$ level, the relative populations increase by more than a factor of four over the energy range of Figure 11, while the $N^{+}=1$ population falls by a factor of two. In contrast to the $\mathrm{N}_{2}$ results, these $\mathrm{CO}^{+}$distributions show essentially no dependence on energy. This difference in the ion rotational distributions for these two isoelectronic systems over such a broad energy range is quite unexpected. However, agreement between the calculated and measured spectra is generally excellent.

To gain insight into the underlying dynamical behavior of these ion 


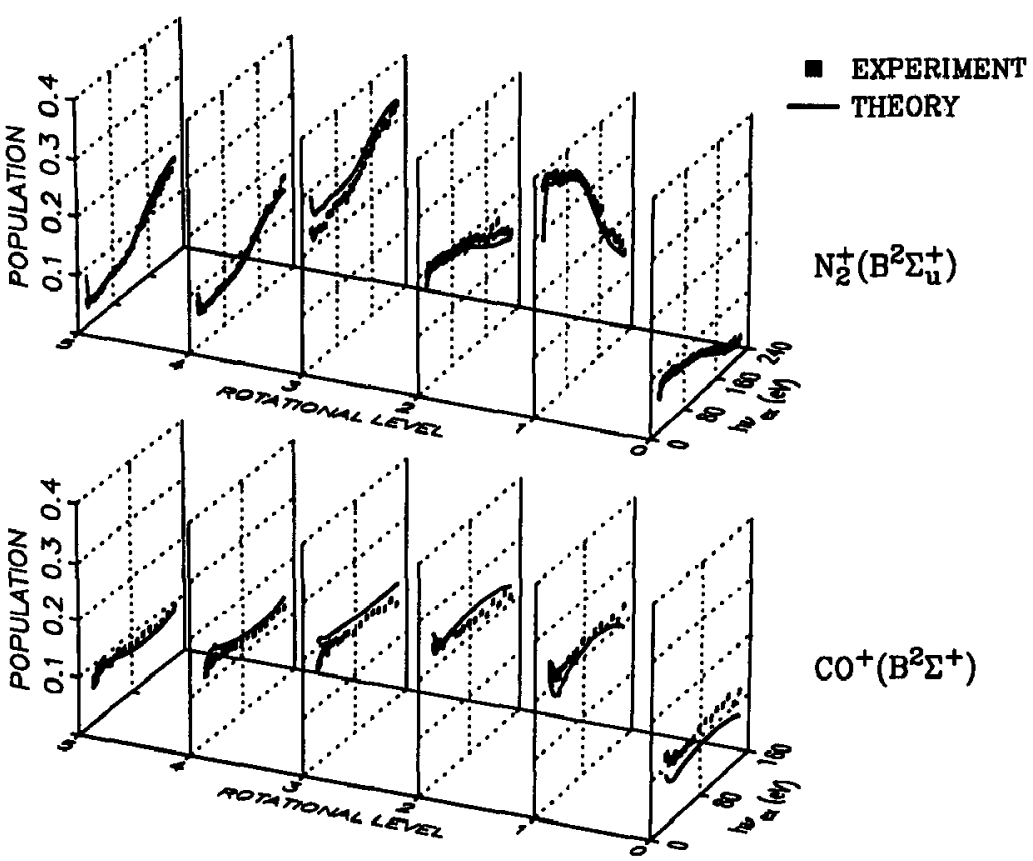

Figure 11 (Top) Calculated and measured rotationally resolved photoion distributions for photoionization of the $2 \sigma_{\mathrm{u}}$ orbital of the ground $X^{1} \Sigma_{\mathrm{g}}^{+}$state of $\mathrm{N}_{2}$ leading to the excited $B^{2} \Sigma_{u}^{+}$state of $\mathrm{N}_{2}^{+}$by synchrotron radiation. (Bottom) Calculated and measured rotationally resolved photoion distributions for photoionization of the $4 \sigma$ orbital of the ground $X^{1} \Sigma^{+}$ state of $\mathrm{CO}$ leading to the excited $B^{2} \Sigma^{+}$state of $\mathrm{CO}^{+}$by synchrotron radiation.

distributions, we examine the dependence of the angular momentum composition of the photoelectron matrix elements on kinetic energy (61). Analysis of the photoelectron matrix elements for photoionization of the $2 \sigma_{\mathrm{u}}$ orbital of $\mathrm{N}_{2}$ reveals that Cooper minima occur in the $d(\ell=2)$ wave at $E_{k} \approx 120 \mathrm{eV}\left(h v_{\mathrm{exc}} \approx 140 \mathrm{eV}\right)$ and in the $g(\ell=4)$ wave at $E_{k} \approx 80 \mathrm{eV}$ $\left(h v_{\mathrm{exc}} \approx 100 \mathrm{eV}\right.$ ) of the $k \sigma_{\mathrm{g}}$ electronic continuum (61). The formation of these Cooper minima depletes the $d$ and $g$ waves and subsequently enhances the $s(\ell=0)$ wave of the $k \sigma_{\mathrm{g}}$ channel, resulting in smaller $\Delta J$ $(\Delta N)$ transitions. Furthermore, the magnitude of the $g$ wave in the $k \sigma_{\mathrm{g}}$ and $k \pi_{\mathrm{g}}$ continua is comparable to those of the $s$ and $d$ waves at larger kinetic energies $(\geqslant 150 \mathrm{eV})$. This behavior reflects the coupling of angular momentum in the photoelectron matrix elements due to torques associated with nonspherical molecular ion potential and cannot be explained on the basis of an atomiclike propensity rule for photoionization of this $2 \sigma_{\mathrm{u}}$ orbital with almost pure $p(90.7 \%)$ character. These higher $\ell$ components 
of photoelectrons, in turn, lead to larger $\Delta N$ transitions. This change from smaller to larger $\Delta N$ transitions due to the Cooper minima, and the contributions of the higher $\ell$ waves provides an explanation of the trend observed in photoion rotational distributions of Figure 11 for $\mathrm{N}_{2}$.

The behavior of photoelectron matrix elements for photoionization of the $4 \sigma$ orbital of the ground state of CO differs significantly from that of $\mathrm{N}_{2}$. There is an $f$-wave shape resonance peaking at $E_{k} \approx 19 \mathrm{eV}$ in the $k \sigma$ continuum and a broad enhancement of the $f \pi$ wave peaking at $E_{k} \approx 30$ $\mathrm{eV}(61)$. This large $\ell=3$ contribution to the photoelectron matrix elements results in larger $\Delta N$ transitions at the lower end of the energy range than expected, while other higher partial waves sustain this trend at higher energies. Thus, the $f$-wave shape resonance in the $k \sigma$ and the enhancement of the $f$ wave in the $k \pi$ channel are responsible for the flat behavior seen in the $\mathrm{CO}^{+}$rotational distributions of Figure 11 . These $f$-wave enhancements are molecular in origin because the $4 \sigma$ orbital of CO, with its $14.6 \%$ $s, 62.3 \% p, 15.7 \% d, 3.1 \% f$, and $2.7 \% g\left(\ell_{0}=4\right)$ character, is expected to lead to dominant $s$ and $d$ waves on the basis of atomiclike propensity rules.

\section{CONCLUSION}

In this article we have reviewed the results of theoretical studies of rotationally resolved photoelectron spectra for several diatomic molecules and for the small polyatomic molecules $\mathrm{H}_{2} \mathrm{O}, \mathrm{H}_{2} \mathrm{CO}$, and $\mathrm{CH}_{3}$. These results and their comparison with measured spectra serve to illustrate both the rich dynamical content of rotationally resolved photoelectron spectra and the effectiveness of a strong interplay between theory and experiment in analysis of such spectra. With the recent development of ZEKE photoelectron spectroscopy, based on PFI of very high Rydberg levels, it is now possible to achieve subwavenumber resolution in molecular photoelectron spectra. Although these ZEKE techniques are limited to measurements of threshold photoionization cross sections, a wide range of molecular ions can be studied. Also, with its broad spectral range, dispersed fluorescence measurements are highly suitable for survey studies that can characterize molecular photoionization dynamics. The present studies indicate that theoretical and computational methods are capable of providing a robust description of key features in such state-resolved spectra.

\section{ACKNOWLEDGMENTS}

This work was supported by grants from the Air Force Office of Scientific Research and the Office of Health and Environmental Research of the US 
Department of Energy. We also acknowledge use of the resources of the Jet Propulsion Laboratory/California Institute of Technology CRAY Y. MP2E/116 Supercomputer.

Any Annual Review chapter, as well as any article cited in an Annual Review chapter, may be purchased from the Annual Reviews Preprints and Reprints service. 1-800-347-8007; 415-259-5017; email: arpr@class.org

1. Grant ER, White MG. 1991. Nature 354: 249-50

2. Pollard JE, Trevor DJ, Reutt JE, Lee YT, Shirley DA. 1982. J. Chem. Phys. 77: $34-46$

3. Pratt ST, Dehmer PM, Dehmer JL. 1984. Chem. Phys. Lett. 105: 28-33

4. Anderson SL, Kubiak GD, Zare RN. 1984. Chem. Phys. Lett. 105: 22-27

5. Peatman W, Wolf FP, Unwin R. 1983. Chem. Phys. Lett. 95: 453-57

6. Pratt ST, Dehmer PM, Dehmer JL. 1990. J. Chem. Phys. 92: 262-74

7. Viswanathan KS, Sekreta E, Reilly JP. 1986. J. Phys. Chem. 90: 5658-61

8. Viswanathan KS, Sekreta E, Davidson ER, Reilly JP. 1986. J. Phys. Chem. 90: 5078-84

9. Allendorf SW, Leahy DJ, Jacobs DC, Zare RN. 1989. J. Chem. Phys. 91: 221634

10. de Beer E, Born M, de Lange CA, Westwood NPC. 1991. Chem. Phys. Lett. 186: 40-46

11. Wang K, Stephens JA, McKoy V, de Beer E, de Lange CA, et al. 1992. $J$. Chem. Phys. 97: 211-21

12. de Beer E, de Lange CA, Stephens JA, Wang K, McKoy V. 1991. J. Chem. Phys. 95: 714-16

13. Milan B, Buma WJ, de Lange CA, Wang K, McKoy V. 1995. J. Chem. Phys. In press

14. Müller-Dethlefs K, Schlag EW. 1991. Annu. Rev. Phys. Chem. 42: 109-36

15. Müller-Dethlefs K, Grant ER, Wang K, McKoy V, Schlag EW. 1995. Adv. Chem. Phys. In press

16. Reiser $G$, Habenicht $W$, MüllerDethlefs K, Schlag EW. 1988. Chem. Phys. Lett. 152: 119-23

17. Tonkyn RG, Winniczek JW, White MG. 1989. Chem. Phys. Lett. 164: 137-42

18. Sander M, Chewter LA, MüllerDethlefs K, Schlag EW. 1987. Phys. Rev. A 36: 4543-46
19. Xie J, Zare RN. 1989. Chem. Phys. Lett. 159: 399-405

20. Choi HC, Rao RM, Mihill AG, Kakar S, Poliakoff ED, et al. 1994. Phys. Rev. Lett. 72: 44-47

21. Pratt ST, McCormack EF, Dehmer JL, Dehmer PM. 1990. J. Chem. Phys. 92; 1831-38

22. Wiedmann RT, Tonkyn RG, Grant ER, White MG. 1991. J. Chem. Phys. 95: 746-53

23. McCormack EF, Pratt ST, Dehmer JL, Dehmer PM. 1990. J. Chem. Phys. 92: 4734-39

24. Braunstein M, McKoy V, Dixit SN, Tonkyn RG, White MG. 1990. J. Chem. Phys. 93: 5345-46

25. Seaton MJ. 1983. Rep. Prog. Phys. 46: 167-257

26. Fano U. 1970. Phys. Rev. A 2: 353-65

27. Fano U, Dill D. 1972. Phys. Rev. A 6: 185-92

28. Dill D. 1972. Phys. Rev. A 6: 160-72

29. Fredin S, Gauyacq D, Horani M, Jungen Ch, Lefevre G, et al. 1987. $\mathrm{Mol}$. Phys. 60: 825-66

30. Child MS, Jungen Ch. 1990. J. Chem. Phys. 93: 7756-66

31. Lucchese RR, Raseev G, McKoy V. 1982. Phys. Rev. A 25: 2572-87

32. Wang K, McKoy V. 1991. J. Chem. Phys. 95; 4977-85

33. Dubs $M$, Bruhlmann U, Huber JR. 1986. J. Chem. Phys. 84: 3106-19

34. Halpern JB, Zacharias H, Wallenstein R. 1980. J. Mol. Spectrosc. 79: 1-30

35. Brown JM, Hougen JT, Huber KP, Johns JWC, Kopps I, et al. 1975. J. Mol. Spectrose. 55: 500-3

36. Edmonds AR. 1974. Angular Momentum in Quantum Mechanics. Princeton, NJ: Princeton Univ. Press

37. Dixit SN, McKoy V. 1986. Chem. Phys. Lett. 128: 49-54

38. Xie J, Zare RN. 1990. J. Chem. Phys. 93: $3033-38$ 


\section{WANG \& McKOY}

39. Raseev G, Cherepkov N. 1990. Phys. Rev. A 42: 3948-62

40. Dixit SN, Lynch DL, McKoy V, Huo WM. 1985. Phys. Rev. A 32: 1267-70

41. Lee MT, Wang K, McKoy V, Machado LE. 1992. J. Chem. Phys. 97: 3905-13

42. Mulliken RS. 1941. Phys. Rev. 59: 87389

43. Wiedmann RT, White MG, Wang K, McKoy V. 1994. J. Chem. Phys. 100: $4738-46$

44. Hunt WJ, Goddard WA. 1969. Chem. Phys. Lett. 3: 414-18

45. Wang K, McKoy V. 1991. J. Chem. Phys. 95: 7872-79

46. Lefebvre-Brion H. 1990. Chem. Phys. Lett. 171: 377-80

47. Wang K, Stephens JA, McKoy V. 1991. J. Chem. Phys. 95: 6456-62

48. Wiedmann RT, White MG, Wang $K$, McKoy V. 1993. J. Chem. Phys. 98: 7673-79

49. Wang K, McKoy V. 1995. J. Phys. Chem. 99: 1643-48

50. Kong W, Hepburn JW. 1995. J. Phys. Chem. 99: 1637-42

51. Haber KS, Jiang Y, Bryant GP, Grant
ER, Lefebvre-Brion H. 1991. Phys. Rev. A 44: 5331-34

52. Tonkyn RG, Wiedmann RT, White MG. 1992. J. Chem. Phys. 96: 3696-701

53. Takahashi $M$, Ozeki $H$, Kimura $K$. 1991. Chem. Phys. Lett. 181: 255-58

54. Reiser G, Müller-Dethlefs K. 1992. J. Phys. Chem. 96: 9-12

55. Tonkyn RG, Wiedmann RT, Grant ER, White MG. 1991. J. Chem. Phys. 95: $7033-40$

56. Lee MT, Wang K, McKoy V, Tonkyn RG, Wiedmann RT, et al. 1992.J. Chem. Phys. 96: 7848-51

57. Lee MT, Wang K, McKoy V. 1992.J. Chem. Phys. 97: 3108-14

58. Gilbert RD, Child MS. 1991. Chem. Phys. Lett. 187: 153-60

59. Blush JA, Chen P, Wiedmann RT, White MG. 1993. J. Chem. Phys. 98: 3557-59

60. Das R, Wu C, Mihill AG, Poliakoff ED, Wang K, et al. 1994. J. Chem. Phys. 101: $5402-5$

61. Das R, Wu C, Mihill AG, Poliakoff ED, Wang K, et al. 1995. J. Phys. Chem. 99: $1741-47$ 


\section{CONTENTS}

SOME SPECTROSCOPIC REMINISCENCES, William Klemperer 1

Chemistry IN THE INTERSTELlar Medium, Eric Herbst 27

SPECTROSCOPY and Quantum Mechanics OF THE HydRogen

Molecular Cation: A Test of Molecular Quantum

Mechanics, Christine A. Leach and Richard E. Moss

Effective Hamiltonian Theory and Molecular Dynamics, Georges Jolicard

Gas-Phase Reactions and Energy Transfer at Very Low

TEMPERATURES, Ian R. Sims and Ian W. M. Smith

109

Heteronuclear NMR Pulse Sequences Applied to Biomolecules, Jeffrey G. Pelton and David E. Wemmer

Theoretical Studies of Polyatomic Biomolecular Reaction

Dynamics, Joel M. Bowman and George C. Schatz

Dynamics and Photochemistry of NeUtral VAN DER WaAls Clusters, Elliot R. Bernstein

Collective Variable Description of Native Protein Dynamics, Steven Hayward and Nobuhiro Go

Time-Dependent Wave Packet Approach to Quantum Reactive SCATTERING, Bret Jackson

High-Resolution Photoelectron Spectroscopy of Molecules, Kwanghsi Wang and Vincent McKoy

EXTERNAL INFRARED REFLECTION ABSORPTION SPECTROMETRY of Monolayer Films at THE AIR-Water InTERFace, Richard Mendelsohn, Joseph W. Brauner, and Arne Gericke

ImAging TeChNiques For the Study OF Chemical ReACTION Dynamics, Albert J. R. Heck and David W. Chandler

Potential Energy Surfaces for Chemical Reactions at Solid

SURfaCES, Barbara J. Garrison and Deepak Srivastava

Algebraic Methods in SPeCtroscopy, Michael E. Kellman 
viii CONTENTS

Photoassociative Spectroscopy of Laser-Cooled Atoms, P. D. Lett, P. S. Julienne, and W. D. Phillips

Design and Regulation of Efficient Photoinduced Electron Transfer in Macromolecular and Photosynthetic Systems, Stefan Franzen and Jean-Louis Martin

NuCleation: Measurements, Theory, and Atmospheric Applications, Ari Laaksonen, Vicente Talanquer, and David W. Oxtoby

ION REACTION DyNamics, James $M$. Farrar

Orientation and Alignment in Reactive Beam Collisions: ReCent Progress, $H$. $J$. Loesch

High-Pressure Structural Transformations in Semiconductor Nanocr ystals, Sarah $H$. Tolbert and A. P. Alivisatos

Femtosecond Pulse-Shaping, Multiple-Pulse Spectroscopy, and Optical Control, Hitoshi Kawashima, Marc M. Wefers and Keith A. Nelson

Salt-Nucleic Acid Interactions, Charles $F$. Anderson and $M$. Thomas Record, Jr.

Density-Functional Theory of the Electronic Structure of Molecules, Robert G. Parr and Weitao Yang

INDEXES

Author Index

Subject Index

753

Cumulative Index of Contributing Authors, Volumes 42-46

765

Cumulative Index of Chapter Titles, Volumes 42-46 